\title{
Fibrillar-Level Strain Gradients Across the Calcified Bone-Cartilage Interface
}

\author{
Waqas Badar ${ }^{1}$, Husna Ali ${ }^{1}$, Olivia N Brooker ${ }^{1}$, E. Newham ${ }^{1}$, Tim Snow ${ }^{2}$, Nicholas J Terrill ${ }^{2}$, \\ Gianluca Tozzi ${ }^{3}$, Peter Fratzl ${ }^{4}$, Martin M Knight ${ }^{1}$, and Himadri S Gupta ${ }^{1}$ \\ ${ }^{1}$ Institute of Bioengineering and School of Engineering and Material Science, Queen Mary University \\ of London, London, E1 4NS, United Kingdom \\ ${ }^{2}$ Harwell Science and Innovation Campus, Diamond Light Source, Harwell, Didcot, OX11 10 0DE, \\ United Kingdom \\ ${ }^{3}$ Zeiss Global Centre, School of Mechanical and Design Engineering, University of Portsmouth, \\ United Kingdom \\ ${ }^{4}$ Department of Biomaterials, Max-Planck-Institute of Colloids and Interfaces, Potsdam \\ Wissenschaftspark, Golm, Germany
}

\begin{abstract}
The bone-cartilage interface (BCI) and underlying calcified plate is a universal feature in diarthrodial joints. The BCI is an important mechanically-graded interface subjected to shear and compressive strains, and changes at the BCI have been linked to osteoarthritis progression. Here we report the existence of a physiological internal strain gradient (pre-strain) across the BCI at the ultrastructural scale of the extracellular matrix constituents, specifically the collagen fibril. We use X-ray scattering that probes changes in the axial periodicity of fibril-level D-stagger of tropocollagen molecules in the matrix fibrils, as a measure of microscopic pre-strain. We find that mineralized collagen nanofibrils in the calcified BCI are in tension pre-strain relative to the underlying trabecular bone. This behaviour contrasts with the previously accepted notion that fibrillar pre-strain (or D-stagger) in collagenous tissues always reduces with mineralization due to reduced hydration and associated swelling pressure. Within the calcified tissue, a finer-scale gradient in pre-strain over $\sim 50 \mu \mathrm{m}$ is likely linked to the tidemark. The increased fibrillar pre-strain at the BCI is linked to prior research reporting large tissuelevel residual strains under compression. The findings may have biomechanical adaptative significance: higher in-built molecular level resilience/damage resistance to physiological compression, and the disruption of the molecular-level pre-strains during remodelling of the BCI may be a potential factor in osteoarthritis-based degeneration.
\end{abstract}

\section{Corresponding Author}

Himadri S. Gupta (h.gupta@qmul.ac.uk) 


\section{Introduction:}

The bone-cartilage unit (BCI) plays a crucial biomechanical role in enabling pain-free articulation and smooth transmission of shear and compressive stresses across diarthrodial joints (1). Structural breakdown and alterations in bone-cartilage cellular communication at the BCI has been proposed as a key early-stage marker for osteoarthritis (2). Osteoarthritis is a debilitating musculoskeletal degenerative condition, with over 300 million people affected (3) and a global prevalence over $20 \%$ for individuals over 40 (4). Development of structural biomarkers for early-stage degeneration in interfaces like the BCI would enable early diagnosis and better therapies (5). As these changes will originate at the molecular- and supramolecular level, understanding the functional design of such interfaces at the nanoscale in both healthy and degenerative conditions is of importance.

The BCI, like other biological interfaces(6), exhibits gradients in both structure and mechanical properties, resulting in gradients in strain during physiological activity. On one side, there is a layer of $\mathrm{AC}$ with depth-dependent gradients in fibre orientation (first described by the Benninghof architecture (7)), type II collagen/proteoglycan content ratios, and hydration (8), and (more recently identified) nanoscale collagen fibrillar pre-strain and intramolecular disorder (9). As shown in Figure 1, collagen fibrils in the AC traverse a tidemark into a layer of calcified cartilage of $(10,11)$, enabling a firm anchorage of the AC cartilage to the underlying subchondral bone. Below the subchondral bone is the more open spongy or trabecular bone network. Scanning X-ray scattering and electron microscopy measurements on the calcified mineral phase in the calcified plate (the combined calcified cartilage and subchondral bone) have revealed gradients in mineral composition (12), crystallinity, and size (10), and recently, differences in the mineral crystallite thickness between healthy and osteoarthritic patients $(11,13)$. The gradient in compressive properties from cartilage (1-10MPa) to subchondral bone (GPa) reduces high interfacial shear stresses which could otherwise lead to cracks and delamination between the softer cartilage and the stiffer bone. Even with graded mechanical properties, physiological loading still induces high apparent strains of up to $5 \%$ in the calcified cartilage interface, measured using DVC (14). Such a combination of high in vivo loading along with gradients across mechanically dissimilar materials requires a structurally optimized design at the material level, to prevent microcrack propagation (15) and enable energy absorption during loading.

At this material level, the extracellular matrix (ECM) of the BCI in articular cartilage, the calcified plate and trabecular bone is essentially a collagen fibrillar composite, with a variable composition of the extrafibrillar phase (Figure 1): glycosaminoglycan (GAG)/proteoglycan(PG) rich in articular cartilage, while predominantly mineralized (carbonated apatite) in the calcified plate and trabecular bone $(16,17)$. Biochemically, articular cartilage and calcified cartilage contain predominantly Type II collagen versus Type I collagen in the subchondral and trabecular bone. Both fibril types exhibit a characteristic D-banding periodicity (D 65-67 nm) which is detectable with methods such as smallangle X-ray scattering (SAXS) $(18,19)$ and atomic force microscopy $(20)$. Shifts in the D-period have been linked to fibril strain in bone, tendon, and cartilage (18,21-23), although recent work highlights the need to consider intra-fibril tilt as well, in tissues like cornea $(22,24)$.

Internal strains and stresses in the fibril phase (both at equilibrium and under loading) are expected to be crucial for structural integrity, joint function and mechanobiological stimuli. The fibril composite in cartilage can be considered as a pre-stressed hydrogel, where swelling pressure exerted by the hydrated GAG/PG is restrained by the collagen fibres leading to tensile pre-strain on the collagen fibrils, identified by an increase in D-period. Recently, our synchrotron X-ray scattering analysis of the ECM nanostructure in the articular cartilage has shown that this fibrillar pre-strain exhibits an increase from the superficial/transitional to the deep zone by $\sim 1 \%$ (corresponding to $\sim 5 \mathrm{MPa}$ ) adjacent 
to the calcified plate in the order of (9). Such nanoscale gradients are associated with the wellestablished micro- and macro-level gradients in fibril orientation from parallel to perpendicular to the joint surface known previously (25). Under compressive loading or synthetic ageing, we demonstrated that these fibrillar gradients in pre-strain are disrupted, indicating the gradients play a mechanically important role in the stabilisation of articular cartilage (9). However, a limitation of the previous study (9) was that the mechanically crucial calcified plate and underlying bone was not considered, as biopsies were taken by cutting the tissue at the BCI, in common with many other studies measuring the mechanical properties of cartilage explants ex-vivo. Indeed, mineralized collagen (as found in the calcified plate and trabecular bone) has usually a lower D-period than unmineralized (as in articular cartilage), which has been explained via loss of water inside the fibril (26-28). The pre-strain thus increases from the transitional/superficial to deep zone cartilage (9), lying adjacent to the underlying mineralized tissue with a putatively lower D-period. The calcified plate is subject to significant tissuelevel and microscale strains in loading (14). However, the corresponding ultrastructural D-period variation in the calcified plate (relative to the articular cartilage and trabecular bone) has not been measured.

This present study aims to measure the gradient in pre-strain in collagen D-period and related ultrastructural properties in the BCI, focusing on the calcified plate, to better characterise this biomechanical interface critical for healthy joint function. A bovine model of the bone-cartilage interface from metacarpophalangeal joints is used $(9,18)$. High brilliance synchrotron X-ray SAXS mapping, in the fast fly-scan modes, allows relatively rapid data acquisition across micro- and macroscale tissue regions. The high X-ray flux at synchrotrons enable localized measurements with a micro-beam, necessary to detect micron-scale variation of nanoscale ECM parameters. We carry out SAXS mapping with multiple (three) spatial resolutions 1) across the entire articular cartilage, calcified plate, and trabecular bone, and 2) focused on the articular cartilage and calcified plate, and 3) a high-resolution mapping of the calcified plate region. Our results will identify whether potentially mechanically important equilibrium or pre-strain gradients exist at the ECM level, helping establish baselines for deviations in osteoarthritis and other musculoskeletal degeneration.

\section{Materials and Methods:}

\section{Sample preparation:}

Bone-cartilage unit (BCU) explants were extracted from a metacarpophalangeal (MCP) joint (Figure 1C), of freshly slaughtered adult bovine steers (16-24 months of age), delivered by the local abattoir. Bovine cartilage was selected for use in the experiment, as it is both widely available, has less variability as compared to human cartilage samples and is the next best biomechanical approximation of human cartilage(29). The intact joints were washed with biological detergent followed by immersion in disinfectant (Chemgene) for 15 minutes (this procedure did not affect the internal cartilage and bone tissue as the joints were intact). Joints were then opened using sterile disposable scalpel blades (Swann Morton) in a biohazard safety cabinet. The metacarpal bone was removed, and two metacarpal condyles were isolated from each joint using a high high-speed saw. Extracting intact bone-cartilage cores required a custom sample-preparation process; a method inspired by the work of Aspden and co-workers was used (30). First, each condyle was placed in a custom-designed 3Dprinted holder with an axis of rotation matching that of the condyle flexion axis. A bench drill (Axminster), with a diamond coated coring drill bit was used to produce cores of $2 \mathrm{~mm}$ diameter, under constant irrigation. The condyle was rotated around the sample-holder axis so that, at each extraction point on the condyle, the drill-face was incident locally normal to the condylar surface; this ensured flat cylindrical samples. The drill was used to core to a $5 \mathrm{~mm}$-depth at five locations on the proximal surface of each condyle. To complete the extraction, the intact condyle was removed from 
the 3D-printed holder, and the reverse face of the condylar section was abraded continuously with a high-speed Dremel rotary tool equipped with a burring-bit, until each core detached. Both drilling systems were used with constant phosphate buffered saline solution (PBS) (Sigma-Aldrich, Poole, UK) irrigation, with the drilling advancing slowly to minimize heat and mechanical damage. Finally, the extracted cores were cut to an equal length of $5 \mathrm{~mm}$ by removing excess $\mathrm{TB}$, using a Buehler IsoMet low-speed saw and a 3D printed sample holder under constant irrigation. Final cores, as shown in Figure 1C, were placed in Eppendorf tubes with Dulbecco's Modified Eagle's Medium - low glucose (DMEM) (Sigma-Aldrich, Poole, UK), and stored at $-20^{\circ} \mathrm{C}$ until the experiments were conducted at Diamond Light Source (DLS).

\section{SAXS measurement protocols:}

SAXS scanning of the BCU were performed at beamline I22 (31), Diamond Light Source (DLS, Harwell Science and Innovation Campus, Didcot, UK). The beam size at the sample was measured to be $20 \mu \mathrm{m}$, beam energy of $14 \mathrm{keV}$ and the sample to detector distance at $5.8 \mathrm{~m}$. The samples were mounted in film cassette windows (Figure 1C), which were sealed using a bilayer Kapton film arrangement with enclosed PBS, to ensure hydration over the duration of the SAXS scan. SAXS scanning was performed with a Pilatus $2 \mathrm{M}$ detector (32) (pixel size $172 \mu \mathrm{m}$; resolution $1475 \times 1679$ pixels) with an exposure time of 1s for each SAXS measurement. SAXS scans were carried out in "fly-scan" mode where the sample-stage moves continuously during the measurement; this ensures rapid data collection whilst still maintaining spatial registration. Three different SAXS scan settings were used:

Full-length scan: To obtain the SAXS data for the full-scale length of the extracted BCU core shown in Figure 1C (extending from AC through the full length of TB), a rectangular 2D area scan of 5mm (vertical $(y)$; distance from joint surface) $\times 0.38 \mathrm{~mm}$ (horizontal $(x)$; distance parallel to joint surface) was performed, with $20 \mu \mathrm{m}$ increments in both $x$ and $y$-direction.

Detailed scan: For the SAXS data focussed on the BCI, a square 2D area scan of $0.4 \mathrm{mmx} 0.4 \mathrm{~mm}$ along the interface was performed with $5 \mu \mathrm{m}$ increments in the $\mathrm{x}$ and $\mathrm{y}$-direction.

Regular scan: Due to the time-intensive nature of the full-length $(\sim 1.5 \mathrm{hrs})$ and detailed $(\sim 1.75 \mathrm{hrs})$, and the need to obtain repeats, SAXS scans of 6 more samples were performed with lower spatial resolution and over a shortened vertical direction, which included the full $\mathrm{AC}$ and $\mathrm{CP}$ tissue but only a part $(1.36 \mathrm{~mm})$ of the $\sim 4 \mathrm{~mm}$ long TB region. Specifically, a rectangular 2D area scan of $2.6 \mathrm{~mm}$ (vertical $(y)) \times 0.8 \mathrm{~mm}$ (horizontal $(x)$ ) was performed with $40 \mu \mathrm{m}$ increments in both $x$ - and $y$ direction.

\section{Data Processing:}

SAXS analysis was carried out via a combination of DAWN (www.dawnsci.org), an Eclipse-based software framework developed at Diamond Light Source(33), with customized model fitting routines (in Python; Anaconda Python distribution from Continuum) applied to the 1D integrated intensity files generated by DAWN. Fibril-level ultrastructural parameters (fibrillar D-period, fibrillar orientation and degree of fibrillar alignment) were extracted from SAXS patterns, via both prior published protocols developed in our group $(9,18)$, and by use of new (non-parametric) methods of intensity peak centre and width estimation.

DAWN Data Reduction: The 2D-rastered maps of 2D SAXS data (1 per array point) for each scan (shown in Figure 1D), were reduced to $2 \mathrm{D}$ arrays of $1 \mathrm{D}$ intensity profiles. The $\operatorname{radial} I(q)$ and azimuthal (angular) $I(\chi)$ files were extracted as a function of scattering vector $(q)$ or azimuthal angle $(\chi)$ depending on integration mode (azimuthal and radial, respectively) shown in Figure 1E, and saved as text files. The Processing perspective in DAWN was used for the batch reduction of the data 
files (33). As well as a mask file specific for the Pilatus detector, which excluded the dead-regions (seen as white strips in Figure 1D) between charge-coupled device (CCD) plates or the beam-stop. $I(q)$ processing for Fibril D-period: Figure 1G shows an $I(q)$ plot from our data with DAWN showing the radial meridional peaks, particularly clear at the $3^{\text {rd }}$ and $5^{\text {th }}$ order diffraction peaks (wavevector range 0.1 to $1.2 \mathrm{~nm}^{-1}$ ). For collagen-specific analysis, the $3^{\text {rd }}$ order meridional peak was used because it is clearly visible in both (uncalcified) cartilage, the calcified plate and in trabecular bone (in contrast to the $5^{\text {th }}$ order peak which is clearly visible in articular cartilage (9) but less clear in calcified tissue). After azimuthal integration on DAWN, the $I(q)$ data sets produced for each scan point were read by custom Python scripts to perform the peak analysis for the desired parameters, as described below:

1) Total SAXS intensity: The total SAXS intensity was calculated from the area under the $I(q)$ curve. Total SAXS intensity, primarily from diffuse scattering, is related to the amount of interfacial area between nanoscale inclusions like mineral platelets and surrounding matrix in bone (34), or between fibrils and the extrafibrillar matrix. Here, we use it to a) distinguish between the bone-cartilage core and surrounding fluid and $b$ ) to distinguish between the calcified and uncalcified tissue regions in the scan, as calcified tissue regions have much higher diffuse SAXS intensity arising from the mineral phase.

2) Collagen peak intensity: The collagen peak intensity is defined as the total area under the $3^{\text {rd }}$ order meridional collagen peak after diffuse background SAXS subtraction. Diffuse SAXS background subtraction is done as per our previous protocols $(9,35)$, by a linear interpolation of the diffuse signal from the left $\left(q=0.27 \mathrm{~nm}^{-1}\right)$ to the right $\left(q=0.3 \mathrm{~nm}^{-1}\right)$ of the $3^{\text {rd }}$ order meridional peak. The collagen peak intensity is related to a) the total amount of collagen fibrils/unit volume present in the scattering volume b) the degree of intrafibrillar order (18) and c) the orientation of the fibril in 3D space (36).

3) Fibril D-period: The fibril D-period arises from the axial periodicity in electron density along the collagen fibril due to the gap-overlap stacking of tropocollagen molecules inside the fibril(37). Shifts in the D-period can be linked to axial fibril strain (e.g. (18,38-40)), and in cartilage, changes in the Dperiod have been interpreted as changes in tensile pre-strain due to variations in swelling and osmotic pressures in the proteoglycan-rich extrafibrillar phase $(9,18)$. For all points in the 2D scan, we set a threshold of 0.025 (arbitrary units) in collagen peak intensity (from 2) above), above which the Dperiod (and associated peak parameters) was estimated. The need for the threshold is because estimating the fibril D-period is only meaningful where there are sufficient collagen fibrils at a point in the scattering volume satisfying the Ewald diffraction condition and generating a clear $3^{\text {rd }}$ order meridional peak.

To estimate D-period, initially a parametric peak fitting approach (starting with Gaussians) was used, following prior work in our group $(9,41)$ and using the lmfit nonlinear least squares fitting package in Python (42). However, we found that there was a pronounced rightward asymmetry in the peak profile, which was not visible in prior work $(9,41)$. The possible structural reasons for this effect (arising from fibre diffraction), and its implications for the measured D-period will be explored and justified in detail in the Discussion. Here, we note only that the peak asymmetry is visible due to the recent upgrade of X-ray optics at the I22 beamline (in 2019) which led to much higher resolution in the peak profile which could not be resolved in earlier work $(9,18)$. Due to the asymmetric peak nature, we use a nonparametric method-of-moments approach, treating the $I(q)$ profiles (with the diffuse linear background intensity subtracted) as continuous distributions around the central wave vector (q) values, to find the parameters centre $(\mu)$ and $\operatorname{sigma}(\sigma)$. The first moment $\left(\mathrm{q}_{\mathrm{FM}}=\mu\right)$, that is the expectation value $\mu$, gave the position of the peak $\left(\mathrm{q}_{0}\right.$ in our prior notation(9)) and the standard deviation gave $\sigma$ (the width of the peak). The following equations were used to calculate the first moment, the second moment and the standard deviation. 


$$
\begin{array}{cc}
\text { First moment } \mathrm{q}_{0} \text { or } \mathrm{q}_{\mathrm{FM}}=\frac{\sum q I_{b}(q)}{\sum I_{b}(q)} & \text { Equation 1 } \\
\text { Second moment, } \mathrm{q}_{\mathrm{SM}}^{2}=\frac{\sum q^{2} I_{b}(q)}{\sum I_{b}(q)} & \text { Equation 2 } \\
\text { Standard deviation, } \sigma=\sqrt{\mathrm{q}^{2} \mathrm{SM}-q_{F M}^{2}} & \text { Equation 3 }
\end{array}
$$

where $q$ and $I_{b}(q)$ are the wave vector and (background corrected) radial intensity values around the 3rd order peak, as shown in Figure 1G. From $q_{0}$ the D-period was calculated as (where $n$ is the peak order, $\mathrm{n}=3$ for the 3 rd order meridional peak):

$$
\mathrm{D}=n \frac{2 \pi}{q_{0}} \quad \text { Equation } 4
$$

To represent the SAXS scans as 2D images, parameters (like D-period, total SAXS intensity and collagen peak intensity) were displayed as a colour-scale bit-map, where the colour value was related to the value of the calculated parameters through a colour-map.

$I(\chi)$ processing for fibril orientation and degree of fibrillar alignment: $1 \mathrm{D}$ azimuthal intensity profiles $\mathrm{I}(\chi)$ at each scan point were generated to obtain parameters characterising the angular collagen fibril distribution - the average fibril orientation $\chi_{0}$, and degree of fibrillar alignment ( $\rho$ : random order $\rightarrow \rho=0$, uniaxial alignment $\rightarrow \rho$ large). In a similar manner to the $I(q)$ analysis, the background diffuse scattering needs to be corrected for. A three-ring subtraction method was used to obtain corrected intensity value, by radially averaging the intensity just outside of the peak and subtracting this average from the radial average on the peak, as described previously by us (43):

\section{Collagen Intensity = Peak intensity $-1 / 2($ inner intensity + outer intensity $)$ Equation 5}

It is noted that across the full azimuthal $0-360^{\circ}$ range, some parts of the detector were blocked due to dead-regions between Si pixel modules or the beam-stop. A restricted range from 0 to $180^{\circ}$ where such blockages were minimized was therefore used. This restricted angular range provides the full information due to the theoretical $\chi \rightarrow \chi+180^{\circ}$ symmetry of the scattering for SAXS (from to the Ewald sphere being approximated as a plane). The corrected intensity peaks, $\mathrm{I}_{\text {corrected }}$ (after background subtraction in Equation 5) were fitted with a Gaussian model with zero baseline using a custom Python program using the lmfit nonlinear fitting package (42), as shown in Figure $\mathbf{1 H}$ and following our prior work $(9,43,44)$.

The $\rho$-parameter was calculated from $\mathrm{I}_{\text {corrected }}$ data by evaluating the Gaussian model fit results, using the following equation

$$
\rho=\left(\mathrm{I}_{\text {corrected_max }}-\mathrm{I}_{\text {corrected_min }}\right) / \mathrm{I}_{\text {corrected_mean }} \quad \text { Equation } 6
$$

where $\mathrm{I}_{\text {corrected_max }}$ and $\mathrm{I}_{\text {corrected_min }}$ are the maximum and minimum values in the fit for $\mathrm{I}_{\text {corrected }}$.

A custom Python script using the matplotlib package(45) was used to display the colour map of the calculated orientation parameters, where the angle of the line shows the fibrillar orientation. Both the length of the line, and the colour value of each pixel corresponds to the degree of fibrillar alignment $(\rho)$, similar to prior work (10). The definition of degree of fibrillar alignment $\rho$ used here differs from the rho-parameter used earlier for degree of mineral particle alignment in bone (46), but has qualitative similarities in that both are used to represent angular anisotropy of nanoparticle/nanofibre alignment and are close to zero for randomly oriented nanoscale inclusions. 
Zonal assignment: To analyse differences in these SAXS parameters across tissue zones, we used scalar measures from the SAXS pattern to classify a point as belonging to 1) articular cartilage (AC), 2) calcified plate (CP), or trabecular bone (TB). Within articular cartilage, we further subcategorized points as arising from the superficial, transitional, or deep zone. First, a threshold of 2.0 [arbitrary units] in total SAXS intensity was used to differentiate between articular cartilage (low total SAXS intensity) and calcified plate (high total SAXS intensity due to mineral scattering). The dense cortical calcified plate and the spongy trabecular bone regions were differentiated based on the vertical $y$ coordinate, as the cylindrical sample geometry leads to a simplified 1D (vertical y) variation in the sample tissue structure, and a visually clear demarcation between the calcified plate and the trabecular bone. For zones within in the articular cartilage region, the degree of fibrillar alignment $\rho$ and direction of fibril orientation $\chi_{0}$ were used as differentiators: points with low $\rho(<2.1)$ with randomly aligned fibrils were assigned to the transitional zone (TZ). Points above the transitional zone and with $\chi_{0}$ around $0^{\circ}$ or $180^{\circ}$ (horizontal) was assigned to the superficial zone (SZ), and points below with $\chi_{0}$ around $90^{\circ}$ were assigned to the deep zone (DZ). The justification for this assignment is the wellknown arcade-like Benninghof structure (7) for the fibril orientation in articular cartilage. For all samples, a 5-color map (SZ/TZ/DZ/CP/TB) of the scans was generated, based on these assignmens, and visually inspected to check that this classification did not result in any artefacts, e.g. an assignment of a point deep inside the trabecular bone to the calcified plate category; all 5-color maps for regions are also shown in Supplementary Figure 1.

Data analysis, fitting and statistical analysis: Statistical comparisons across zones were carried out by 1-way ANOVA using R (www.r-project.org), followed by Tukey's honestly significant difference (HSD) pair-wise tests. Custom Python scripts (Anaconda Individual Edition; www.anaconda.com) using the packages Imfit (42)and matplotlib (45) were used for the analysis of $I(q)$ and $I(\chi)$ profiles created in DAWN, along with Microsoft Excel.

\section{Results:}

In the following, we present quantitative 2D mapping and zonal analysis of ultrastructural SAXSbased parameters across the bone-cartilage interface at different length-scales. As shown in Figure 1, the different tissue regions are classified into five zones from the surface of the joint on down - in articular cartilage, the superficial (SZ), transitional (TZ) and deep (DZ) zones - and in the calcified tissue, the dense calcified plate (CP: a mix of calcified cartilage and subchondral bone) and the trabecular bone (TB).

Figure 2 shows, via 2D colour-maps of the SAXS parameters, the spatial variation of nanostructural parameters (D-periodicity, total SAXS intensity, collagen peak intensity and $\rho$ ) across the entire BCU (the long scan referenced in Materials and Methods). The 4-color map (no superficial zone was detected in the long scan) shows the expected spatial classification of the tissue into transitional and deep zones inside articular cartilage, and the calcified plate and trabecular bone. The D-period exhibits two clear and unexpected features: i) a gradient of increasing D-period from the surface of the joint to the deep zone in articular cartilage, which is maintained in the calcified plate, followed by ii) a distinctly lower D-period range across the underlying trabecular (where no spatial gradient is seen). It is noted that the D-period in the calcified plate is higher than the trabecular bone despite both being comprised of mineralized collagen.

Figure 2 also shows that the total SAXS intensity is low in AC, shows a clear high band in the CP, due to strong SAXS scattering contrast from the mineral relative to the surrounding organic phase, 
and exhibits a patchy cellular structure in the TB, reflective of the porous mineralized tissue structure in TB. The collagen peak intensity is largest in the cartilage DZ, and decreases when going into mineralized tissue (CP and TB). Notably, just below the $\mathrm{CP}$ there is a region of $\sim 1 \mathrm{~mm}$ where there is minimal collagen peak intensity despite patches of high total SAXS intensity. Such a feature may arise due to tilting of fibrils out of the vertical plane, because of which the meridional peaks do not satisfy the Ewald diffraction condition. The variation of the degree of alignment $\rho$ is as expected: higher $\rho$ values in DZ and CP as compared to TZ and TB.

The qualitative differences in D-period and $\rho$ visible in Figure 2 are statistically significant between the different tissue zones. Figure 3 shows box plots (with error-bars) for D-period, $\rho$ and $\chi_{0}$ across the 4 zones in the full-length scan in Figure 2. The D-period increases from the TZ to the CP, followed by a large drop in the TB (which also has the largest intra-zone variation). 1-way ANOVA tests of difference in D-period across zones shows the zone effect is extremely significant overall $(p<0.001$; Supplementary Table 1); moreover, post-hoc tests show extremely significant differences $(p<0.001)$ present for each pairwise comparison of zones. The degree of alignment $\rho$ is also significantly zonedependent $(\mathrm{p}<0.001)$, with higher $\rho$ in DZ and CP, and lower $\rho$ in TZ and TB. All pairwise differences are extremely significant $(\mathrm{p}<0.001)$, except for between the adjacent $\mathrm{DZ}$ and $\mathrm{CP}$ regions, and the TZ and TB regions. The average fibril orientation $\chi_{0}$ shows no significant difference across regions $(\mathrm{p}>0.05)$. The error bars show there is a wide range of fibril orientations in the cartilage $\mathrm{TZ}$ and in the porous TB region, as expected, but a much narrower distribution in the DZ and CP.

2D maps from a representative example of the regular SAXS scans (as defined in Materials and Methods) are shown in Figures 4 and 5. The regular scans are very similar to the long-scans reported in Figure 2 but are taken over a shorter vertical extent to reduce scan time and enable repeat measurements on different BCU cores. In this example (and for 5 out of the 6 in this set of scans), the thin SZ is visible as well. The SZ, characterised by fibrils oriented parallel to the joint surface, is most easily identified by fibre direction (in Figure 5) coupled with a higher degree of alignment $\rho$ than in the adjacent TZ. It is clear from Figures $\mathbf{4}$ and $\mathbf{5}$ that the gradient of D-period is present, with the qualitative variation of the other parameters also the same as for the single high-resolution SAXS scan in Figure 2. The colour map for the orientation and $\rho$ for the regular scan shows that the orientation follows the well-established ultrastructure of fibres, with SZ fibrils aligning along the horizontal plane (around $0^{\circ} / 180^{\circ}$ angle), no preferred direction in $\mathrm{TZ}$, fibrils in the $\mathrm{DZ}$ and $\mathrm{CP}$ aligning in the vertical plane (around $90^{\circ}$ angle) and no preferred direction in TB (Figure 5). Quantitatively, bar-plots of the zonal variation in D-period, $\rho$ and $\chi_{0}$ across BCU for 6 samples (Figure 6) show the repeatability of the trends in Figure 3. Out of 6 samples, 1 sample's SZ was missing (it might have damaged during sample preparation). The statistical differences between the zones are the same as in Figure 3; with the SZ zone parameters are added. The D-period from the SZ is lower than the TZ, DZ and CP, and slightly higher than in the TB. For the fibril orientation $\chi_{0}$, the orientation in the $\mathrm{SZ}$ is around $0^{\circ}$ and around $90^{\circ}$ in $\mathrm{DZ}$ and $\mathrm{CP}$, with no preferred orientation and with largest variation in $\mathrm{TZ}$ and $\mathrm{TB}$.

Since the zonal variation in D-period is small (e.g., $\sim 65.2 \mathrm{~nm}$ to $66.0 \mathrm{~nm}$ in Figure 6) as is often the case in collagenous tissues $(18,47)$, it is a reasonable question as to how significant these changes are, and whether our new non-parametric method of peak estimation may be artefactually influencing the difference. First, we note that the magnitude of the effect $(\sim 1 \%)$ is comparable to the fibril-level strains experience in bone under deformation to fracture (43). To further clarify this, we plot $I(q)$ profiles, with and without diffuse SAXS background subtraction, from each of the 5 different zones in an example BCU (Figure 7) to see how evident these peak-profile differences are in the original data 
itself. It is clear, especially from the background subtracted data (Figure 7B), that there are distinct changes in peak position and shape across the zones; most notably, the right-shifted $I(q)$ for TB demonstrates the lowered D-period seen in the colour-plots in Figure $\mathbf{2}$ and $\mathbf{4}$ and quantified in Figures 3 and 5. Figure 7B also shows that in AC, the total SAXS intensity due to the collagen peak alone (excluding the diffuse SAXS scattering) is maximum in the DZ, and least in the SZ. This effect may arise due to a combination of varying collagen concentration as well as degree of fibrillar ordering (higher ordering corresponds to higher SAXS peak intensity $(9,47)$ ). Interestingly, the collagen peak height is also larger in $\mathrm{CP}$ than in the TB region.

To map out the ultrastructural variation of the fibril parameters at the calcified interface itself, Figure 8 shows the high-resolution colour maps with $5 \mu \mathrm{m}$ spacing. Laterally-averaged line-profiles of the same data are shown in Figure 10, to more clearly shown the spatial variation with distance from the $\mathrm{AC} / \mathrm{CP}$ interface. The location at the top of the images corresponds to the transition from unmineralized DZ of AC to the CP, evidenced by the lower total SAXS intensity coupled with the high collagen peak intensity. As indicated by the arrows in Figure 10A, over a region of about $70 \mu \mathrm{m}$, the D-period is observed to increase from $\sim 65.4 \mathrm{~nm}$ to $65.8 \mathrm{~nm}(\sim 0.6 \%$ increase) (dashed line a1), following which the D-period is approximately constant over $\sim 70 \mu \mathrm{m}$ (dashed line a2). The D-period then exhibits a decrease over $\sim 170 \mu \mathrm{m}$ to $\sim 65 \mathrm{~nm}$ (dashed line a3), following which the trend stabilises, which corresponds to reaching the TB region.

Interestingly, on comparing Figure 10A-B, the D-period variations show different correlations with the degree of fibrillar alignment $\rho$, depending on where one looks in the BCI. For the transition from $\mathrm{AC}$ to $\mathrm{CP}$ (on the left in Figure 10A-B), the increase in D-period is accompanied by a decrease in $\rho$ by about 20\% (dashed lines a1 and b1). On the right-hand side in Figure 10A-B (also indicated by the region "SCB" in Figure 9), there is a transition to lower $\rho$ (dashed line b3), followed by further decline as the TB region is approached (dashed line b4). In contrast to the AC/CP transition behaviour, on the right-hand side (CP/TB transition), the D-period decreases with reduction in $\rho$ (dash lines a3 and b3). On the left-hand side in Figure 10C, orientation of the fibrils is predominantly perpendicular to the $\mathrm{AC} / \mathrm{CP}$ interface $\left(\chi_{0}=90^{\circ}\right)$, which is a well-known "anchoring" type of architecture for the uncalcified AC to adhere to the SCB. The variance in orientation is less toward AC/CP interface (evidenced by a more homogeneous colour distribution as shown in Figure 9B). On moving toward the TB-region, we see a trend to wider ranges of fibril orientations $\chi_{0}$, coupled with overall lower degree of alignment $\rho$, which is low in TB.

\section{Discussion:}

In summary, the main new findings in this paper are:

- Existence of a positive gradient in fibrillar D-period (linked to fibril pre-strain), extending across both uncalcified articular cartilage (shown by us before (9)) and in the underlying $\mathrm{CP}$, i.e. across the BCI.

- A reduction of the pre-strain in the underlying TB to the values characteristic for Type I mineralized collagen.

- A finer-scale modulation of this gradient at the DZ/CP interface, with high-resolution scans showing a short-length scale $(\sim 80 \mu \mathrm{m})$ drop and increase in D-period.

- Highly significant differences in D-period across all zones, and in the degree of alignment $\rho$ across all zones except between adjacent DZ/CP and spatially separated SZ/TB. 
- Evidence of two distinct regions with altered nanostructure inside the CP, from both fibrillar alignment and pre-strain.

The increased fibril D-period in the $\mathrm{CP} / \mathrm{SCB}$ is surprising, when considering the well-established fact $(28,48)$ that the D-period for mineralized collagen in bone (for example) is consistently lower than hydrated unmineralized collagen. Mineralisation inside and around the fibrils has been linked to dehydration of collagen $(26,27)$, which leads to a lower D-period $(\sim 65 \mathrm{~nm})$. The reasons for the reduction could include axial shrinkage (26) or a change in molecular tilt (24). However, biochemical differences between Type I (bone) and II collagen (calcified cartilage) may couple with altered intraand interfibrillar mineralisation patterns in CC leading to a larger D-period. In contrast, the D-period of $\sim 65 \mathrm{~nm}$ for TB is consistent with previous studies of Type I mineralized collagen (16). It is noted that calling the changes in D-period as changes in pre-strain is an approximation, since it assumes that the fibrils have similar zero-stress D-periods (or reference states) across the tissue types (AC, CP and $\mathrm{SCB})$. Further, the change in pre-strain may be associated with alterations in molecular level tilt $(22,24)$ or ordering of tropocollagen molecules in the fibril $(18)$, which are intrafibrillar mechanisms we do not consider here.

The higher resolution of the SAXS configuration compared to previous configurations $(9,18)$ means some features like the peak asymmetry are visible where before they were hidden by beam divergence. We believe the finite fibre diameter is influencing this asymmetry via a fibre-diffraction effect (49), conceptually sketched in Figure 11B. Fibrils with axial electron-density periodicity of D and radii $R$ exhibit a set of parallel ellipsoidal layer-line reflections (spacing $2 \pi / \mathrm{D}$ ), with finite width $\left(w_{p}\right)$ proportional to $1 / R$ perpendicular to the fibril axis. Thinner fibrils will have greater $w_{p}$ (e.g. case 3 (thin fibril) vs case 1 (thick fibril) in Figure 11B). The polar azimuthal (circular) average of flat ellipsoids parallel to the $\mathrm{q}_{\mathrm{x}}$ axis thus leads to a rightward skew asymmetry of the integrated peak (simulated data shown in Fig 11B, centre), as the tails of the ellipsoids contribute to larger wavevector values in the azimuthal $(\chi)$ integration. As a result, the measured D-period (via weighted moments) underestimates the true D-period (as $\left.\mathrm{q}_{3}=6 \pi / \mathrm{D}\right)$, with an increasing skew correlated to a reduced measured D-period. Figure 7 shows that (especially in cartilage) there is pronounced rightward skew of the I(q) peak. As a result, the estimated D-period via our nonparametric method is likely an underestimate in these regions, and the pre-strain level in both the AC- and CP-regions are even larger than estimated here. Future work will include concurrent fitting the azimuthal and radial intensity profiles, to independently determine $\mathrm{w}_{\mathrm{p}}$ as well as D, thus enabling a point-wise estimation of this effect. For the current results, we note only that the difference between the D-period of the articular cartilage/calcified plate (Type-II collagen fibrils) and the trabecular bone (Type-I collagen fibrils) is maintained.

The finer-structure gradient at the BCI interface, and the colour-contrasts in Figures 8 and 9, are intriguing and we believe are linked to the two-phase CC and cortical subchondral bone (SCB) materials comprising the $\mathrm{CP}$. The length scale of $\sim 70 \mu \mathrm{m}$ over which the D-period rises from a local minimum right at the DZ/CP interface is similar to previously reported thicknesses of the tidemark (10), and suggests a compliant, less pre-strained region right at the interface. The more homogeneous fibrillar alignment $(\rho)$ in the upper half of the CP in Figure 9A versus the more heterogeneous distribution further down suggests a division between $\mathrm{CC}$ (toward the $\mathrm{AC}$ ) and the SCB (toward the TB). Assuming this assignment, our results demonstrate that the high degree of fibrillar alignment and uniform orientation in the CC (as shown earlier $(10,50,51)$ ) is coupled with a rapid rise in fibril Dperiod which reaches $\sim 65.8 \mathrm{~nm}$ (typical of uncalcified cartilage (9)) and remains constant for $\sim 50 \mu \mathrm{m}$ 
in the $\mathrm{CC}$ and a further 50 microns in the $\mathrm{SCB}$, followed by a decrease over $\sim 100 \mu \mathrm{m}$ in the SCB down to typical $\sim 65 \mathrm{~nm}$ values in the TB .

We speculate that the increased tensile fibril-level pre-strain in the calcified plate (CP; Figures $\mathbf{2}$ and 4) has a biomechanical function related to increased elastic energy storage under compression. In the CP, stiff fibrils are oriented parallel to the compressive loading direction (as shown earlier, e.g. (10)) and in a state of tensile pre-strain relative to the fibrils in TB. On mechanical loading, under compression, the pre-stretched fibrils can compress down from the pre-strain level down to zero, before compressive failure mechanisms like buckling start becoming operative. This mechanism is supported by prior work on patellar joints showing large residual strains in the calcified interface (52), and is shown schematically in Figure 11. This schematic Figure shows how the zones of high nanoscale fibrillar pre-strain (from this work) in the calcified plate correlate closely with the high microscale residual compressive strains at the BCI after compressive loading (measured with digital volume correlation methods on tomographic (CT) images (52)). A limitation of this comparison is that the joint analysed in (52) is the bovine patella, whereas here we have tested the bovine metacarpophalangeal joint. Nevertheless, it can be speculated that this natural gradient in ultrastructural tissue properties may enable absorption (and release) of significant stored elastic energy in regular biomechanical loading, with a material energy density of $1 / 2 \mathrm{E} \varepsilon^{2}$ where $\mathrm{E}$ is the tissue elastic modulus of E 10-20 GPa (52), and $\varepsilon$ the fibril pre-strain level of $\sim 1.5 \%$. Indeed, disruption of this ECM material-level energy absorption mechanism due to tidemark duplication in OA could be a biomarker-level indicator of OA progression.

Turning to the limitations of our work, a basic technical drawback is the use of 2D SAXS mapping of what is intrinsically a 3D cylindrical tissue object. Nevertheless, we mitigate these effects by choosing a cylindrical sample geometry, with relatively tissue homogeneity along the X-ray beam direction, coupled with a limited lateral width across the scan versus the height (high aspect ratio). Further, the variations we are interested in are along the length of the sample, rather than through the thickness, and are thus relatively insensitive to such 3D effects. For full-scale 3D reconstruction of the depthdependent nanostructure, SAXS tensor tomography $(36,53)$ will be essential. Secondly, we limit ourselves to static scanning (no in situ mechanical loading) and leave spatial mapping of fibrillar mechanics under loading to future work. Thirdly, we have limited our sampling to a specific condylar anatomical site in the metacarpophalangeal joint and given that in-vivo strains vary across the joint, it would be interesting to test if nanomechanical parameters like pre-strain shows a correlation with varying physiological force levels across the joint. Lastly, we have not yet considered changes in OA and ageing, to establish a baseline characterisation of the normal BCI in an animal model; a recent study (11) has shown that nanoscale mineral particle thickness is significantly different in OA versus normal human patients and investigating in situ mechanical response in aged human cartilage would no doubt be of importance.

The use of scalar measures like SAXS intensity along with orientational information like fibrillar alignment $\rho$ and direction $\chi_{0}$ enables us to classify tissue location based on properties of the oriented collagenous ECM (as shown in Figures 2B and 4B). The method allows discrimination within the AC (superficial, transitional, and deep zones) as well as between the AC, CP/SCB and TB regions. More advanced machine-learning classification methods have been used on the diffuse SAXS signal enabling distinction of the CP and SCB as well (11); it would be of interest to apply such methods to the current data in future analysis. The nonparametric method for peak estimation arises from the pronounced peak asymmetry and the likely limits of linear background interpolation without a model. 
Recent analysis on pericardium collagen using step-function scattering models may, similarly, point the way to a the better analytical fitting of the peak profiles (54). Nevertheless, the clear distinction between the meridional peaks (particularly the peak positions of the TB viz the $\mathrm{CP} / \mathrm{SCB}$ region) is visible in the raw data itself (Figure 7) and will likely not be affected by such further analyses.

In summary, we have demonstrated the existence of a multiscale gradient in the fibrillar pre-strain across the bone-cartilage interface in bovine joints, along with an elevated pre-strain mainly in the CC zone. The elevated pre-strain in the $\mathrm{CP}$ contrasts with the lower values in the underlying TB. Investigating the link between this nanoscale ECM structural change and the in-vivo biological growth dynamics would be interesting. Further, given that the BCI is subjected to considerable shear strains during loading (14), and disruptions to the interface have been implicated in OA progression $(55,56)$, understanding how the ECM architecture of the interface is designed to resist physiological loading, and how it may be altered in injury, ageing and musculoskeletal degeneration would be worth investigating to both understand the process and as well as to define better structural biomarkers of joint degeneration.

\section{Acknowledgements}

HSG (BB/R003610/1, EP/V011235/1) and NJT (BB/R003610/1, EP/V011235/1) thank the BBSRC and EPSRC for research grant funding. HSG thanks MRC (MR/R025673/1) for funding. We thank Diamond Light Source (DLS) for the generous award of synchrotron beamtime (SM25602-2). 


\section{Figures}

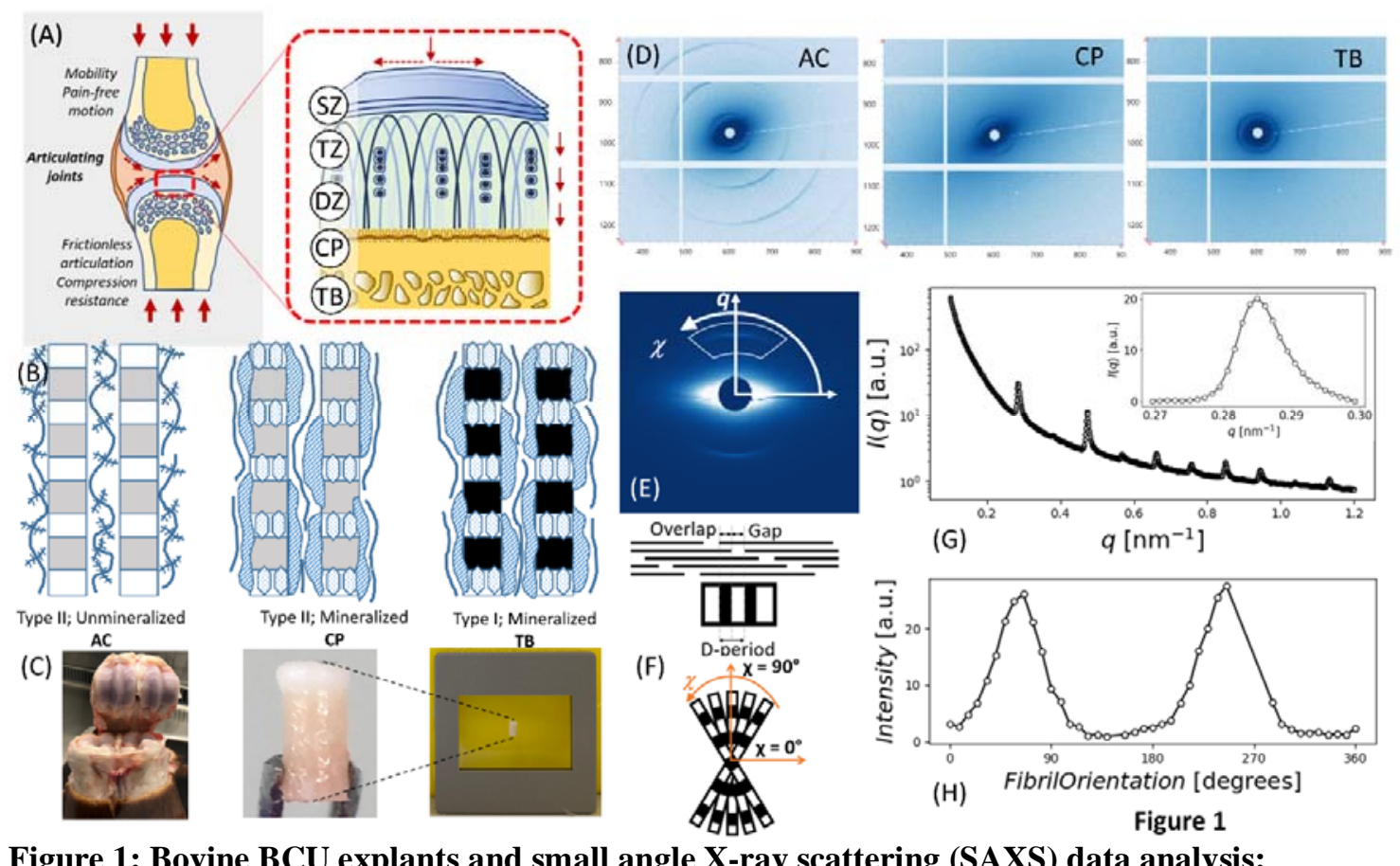

(A) Schematic of joint structure showing the collagen fibril orientation from parallel to the articulating surface in the superficial zone, to vertical in the deep zone and calcified plate. Dashed arrows indicate, schematically, the directions of shear (horizontal) and compressive (vertical) stresses. Abbreviations for region classification: SZ: Superficial zone; TZ: transitional zone; DZ: deep zone: CP: calcified plate, TB: trabecular bone (B) Fibrillar microstructure in AC, CP, and TB zones. Type II collagen fibrils are found in $\mathrm{AC}$ and $\mathrm{CP}$ with mineral particles growing in the gap regions of the $\mathrm{CP}$, whereas type I is found in TB. (C) Bone-cartilage unit (BCU) cores of $5 \mathrm{~mm}$ length and $2 \mathrm{~mm}$ diameter extracted from bovine metacarpophalangeal joints and placed in cassette holder for SAXS scanning while kept hydrated. (D) 2D SAXS patterns from AC, CP, and TB. Low diffuse small-angle X-ray scattering (SAXS) with clear peaks and oriented ellipse in AC, high diffuse SAXS with average peaks and oriented ellipse in CP and high diffuse SAXS with average peaks and no orientation for ellipse in TB. (E) Azimuthal and radial integration to get the $\mathrm{I}(\mathrm{q})$ and $\mathrm{I}(\chi)$ profiles to infer nano structural parameters (D-period, fibrillar orientation and degree of alignment). (F) Fibril schematic: Collagen Dperiod, characterised by the repeating gap-overlap regions causes by the staggered fibril stacking and fibrillar orientation. (G) I(q) plot, showing peaks due to collagen D-period, particularly clear at the $3^{\text {rd }}$ and $5^{\text {th }}$ order after azimuthally integration $\left(0-360^{\circ}\right)$ around the diffraction pattern for the q-radial range $0.1-1.2 \mathrm{~nm}^{-1}$. Right top plot is $3^{\text {rd }}$ order collagen peak after background subtraction. $(\mathrm{H}) \mathrm{I}(\chi)$ profiles after corrected intensity with Gaussian model fitting to the peak. Gaussian fitting was used to infer parameters (fibril orientation and $\rho$ ). 


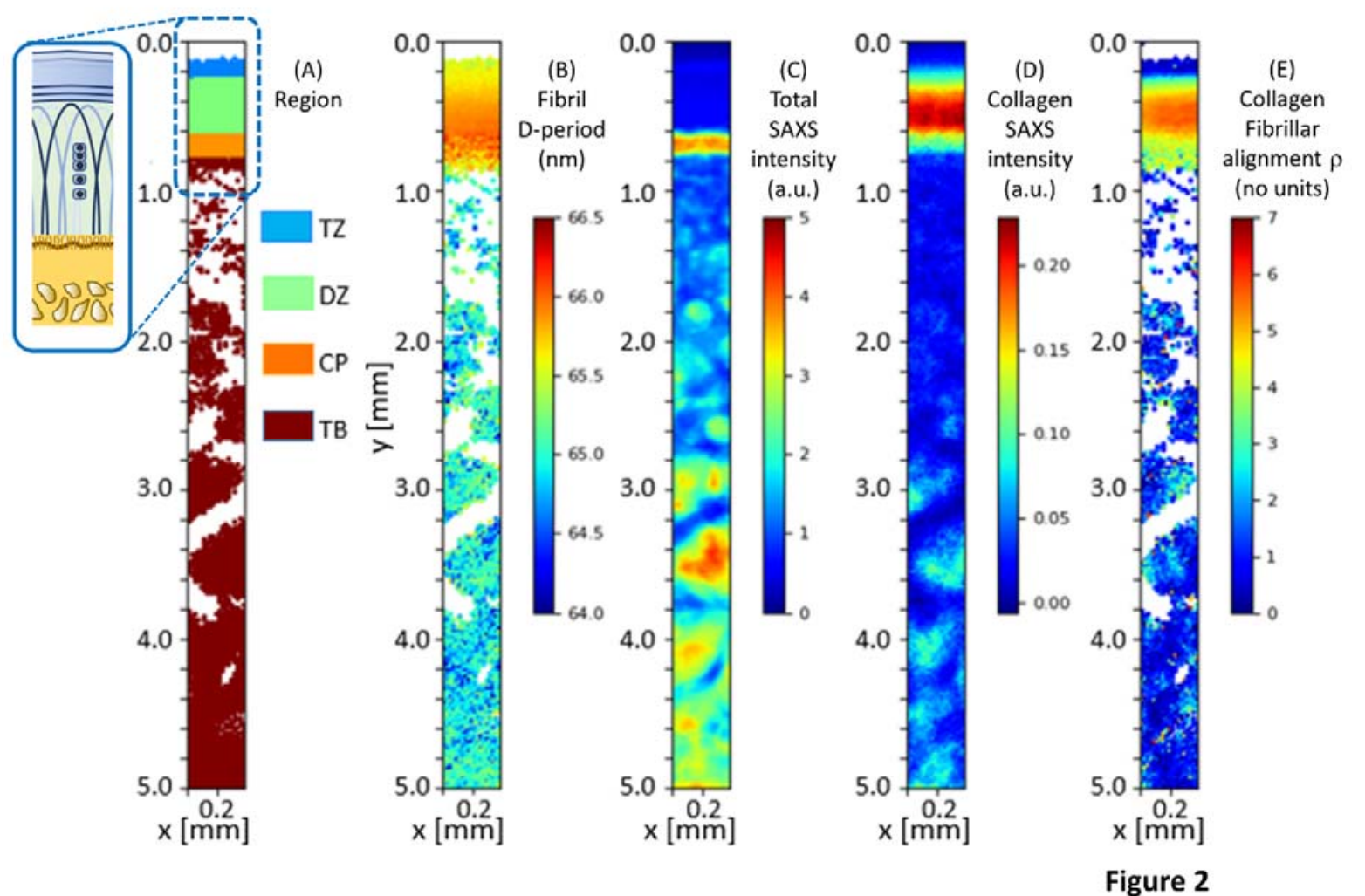

Figure 2: Colour map of the depth-wise variation in SAXS derived parameters across bovine BCU core of $5 \mathrm{~mm}$ length and $2 \mathrm{~mm}$ diameter for full-length scan. SAXS scanning performed with step size of 20 microns across $0.38 \mathrm{~mm}$ width and $5 \mathrm{~mm}$ length of the sample. Note that in this sample we were unable to resolve the thin superficial zone at the top. Colour plots display: (A) D-period (nm), (B) Region (abbreviations defined in Figure 1A), (C) Total SAXS intensity (a.u.) where areas of high intensity correspond with mineral dense regions, (D) Total diffraction intensity from the backgroundcorrected meridional collagen peak intensity (a.u.), where areas of high intensity correspond with the $\mathrm{AC}$ and (E) $\rho$ (a.u.). 

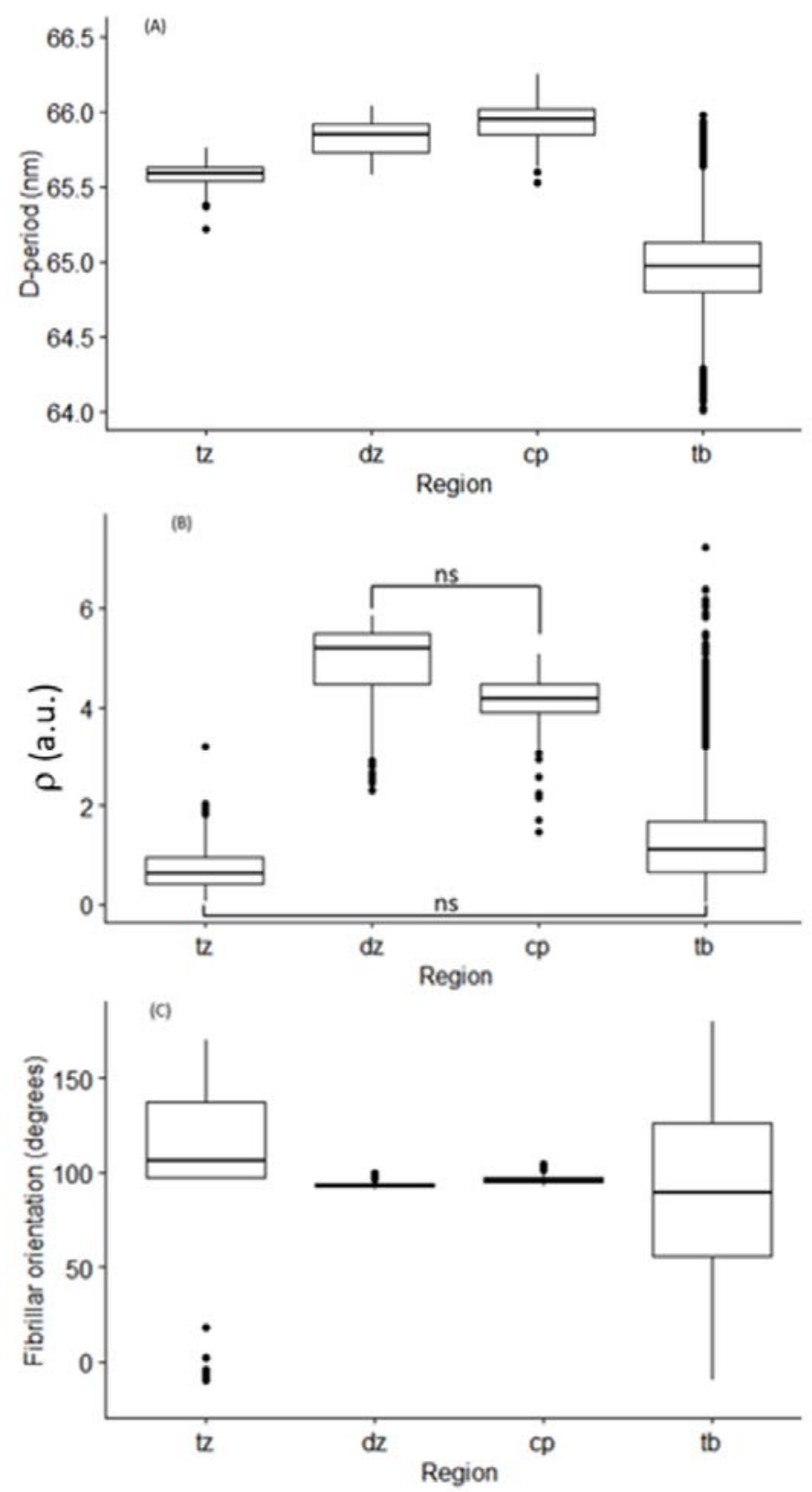

Figure 3

Figure 3: Box plots for nanoscale parameter variation across BCU (A) D-period (B) Degree of fibrillar alignment $(\rho)$ and $(C)$ Fibrillar orientation for full length scan. There is significant difference between the D-period values between the regions, with the lowest value in the TB. Collagen fibrillar orientation in deep zone (DZ) and calcified plate (CP) is around $90^{\circ}$ (perpendicular to the articulating surface), whereas there is no preferred fibrillar orientation in transitional zone (TZ) and trabecular bone (TB). However, the fibril orientation shows no significant difference across regions ( $>0.05) . \rho$ parameter shows that there is a higher degree of fibrillar alignment in $\mathrm{DZ}$ and $\mathrm{CP}$ in comparison to $\mathrm{TZ}$ and TB. The non-significant difference between the regions for $\rho$ parameter is shown in the figure as "ns". 


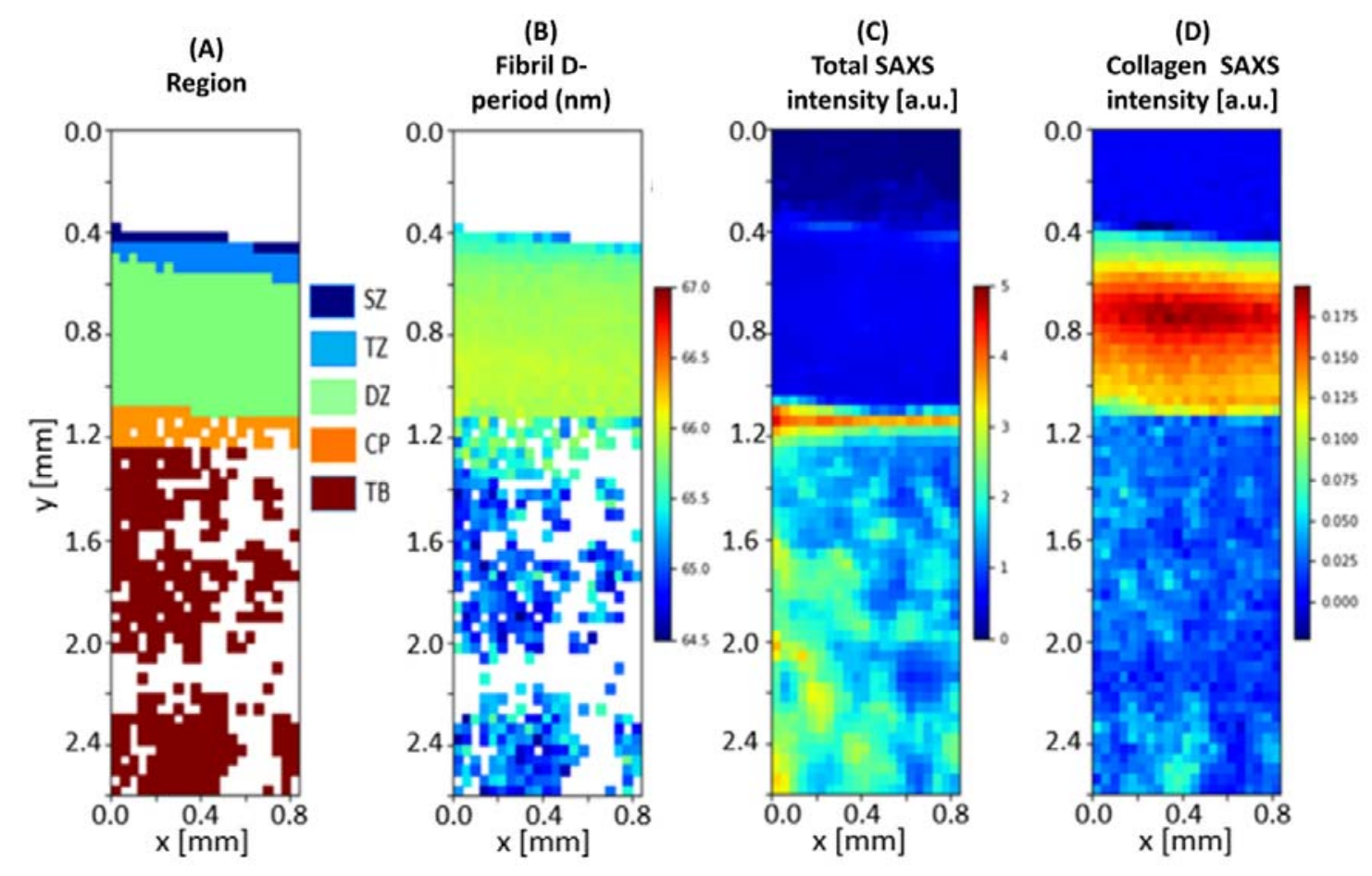

Figure 4

Figure 4: Colour plots display for 2D SAXS scan on a bone-cartilage unit (BCU) with reduced vertical extent compared to Figure 2 (regular scan' $2.6 \mathrm{~mm} \times 0.8 \mathrm{~mm}$ ) (A) Region, with the acronyms following the region classification in Figure 1A, (B) Fibrillar D-period (nm), (C) Total SAXS intensity (a.u.) and (D) Total diffraction intensity from the background-corrected meridional collagen peak intensity (a.u.). The plots show the variation in these nano structural parameters across the BCU, with higher magnification than in Figure 2. 


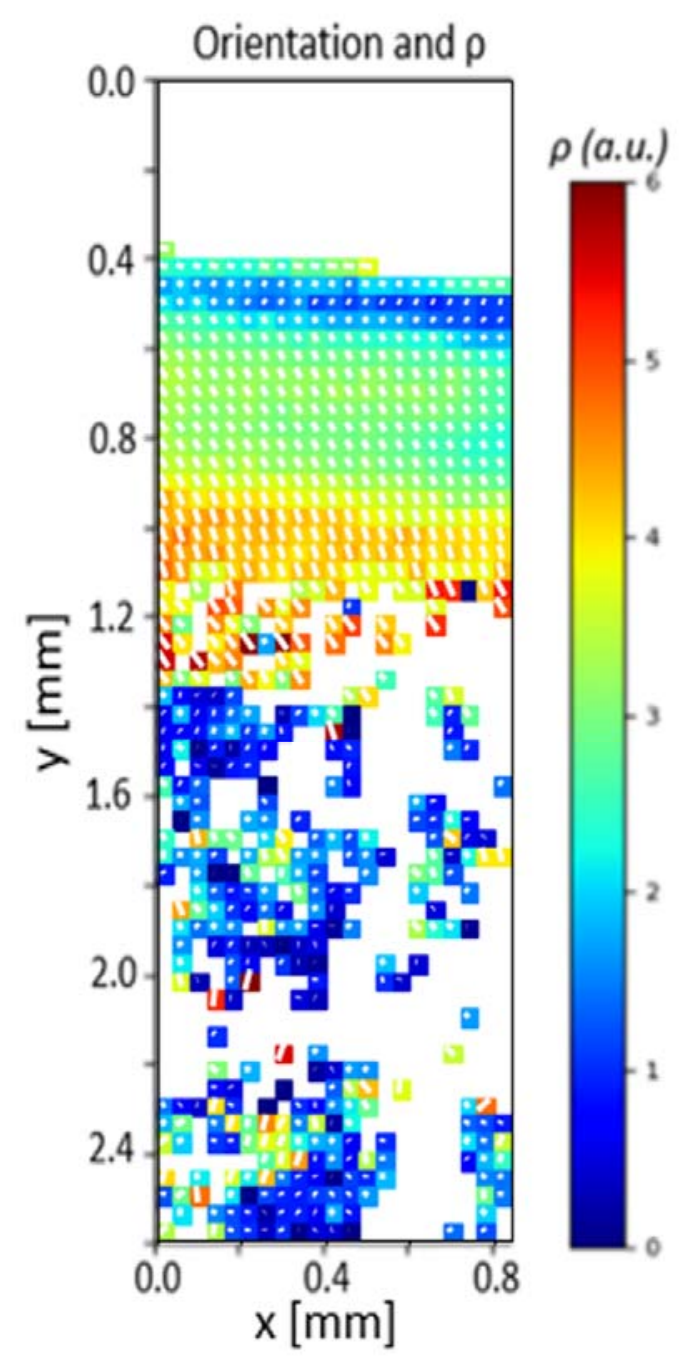

\section{Figure 5}

Figure 5: Colour map of the regular scan for collagen fibril orientation (degrees) and $\rho$ (a.u.) parameter, where the length of the white line and the colour scale corresponds to $\rho$, and the direction of the white line indicates the fibrillar orientation across BCU. There is a clear increase in the degree of fibrillar alignment at the $\mathrm{BCI}$, with the fibrillar orientation perpendicular to articulating surface. However, there is random orientation with lower degree of alignment in TB. 

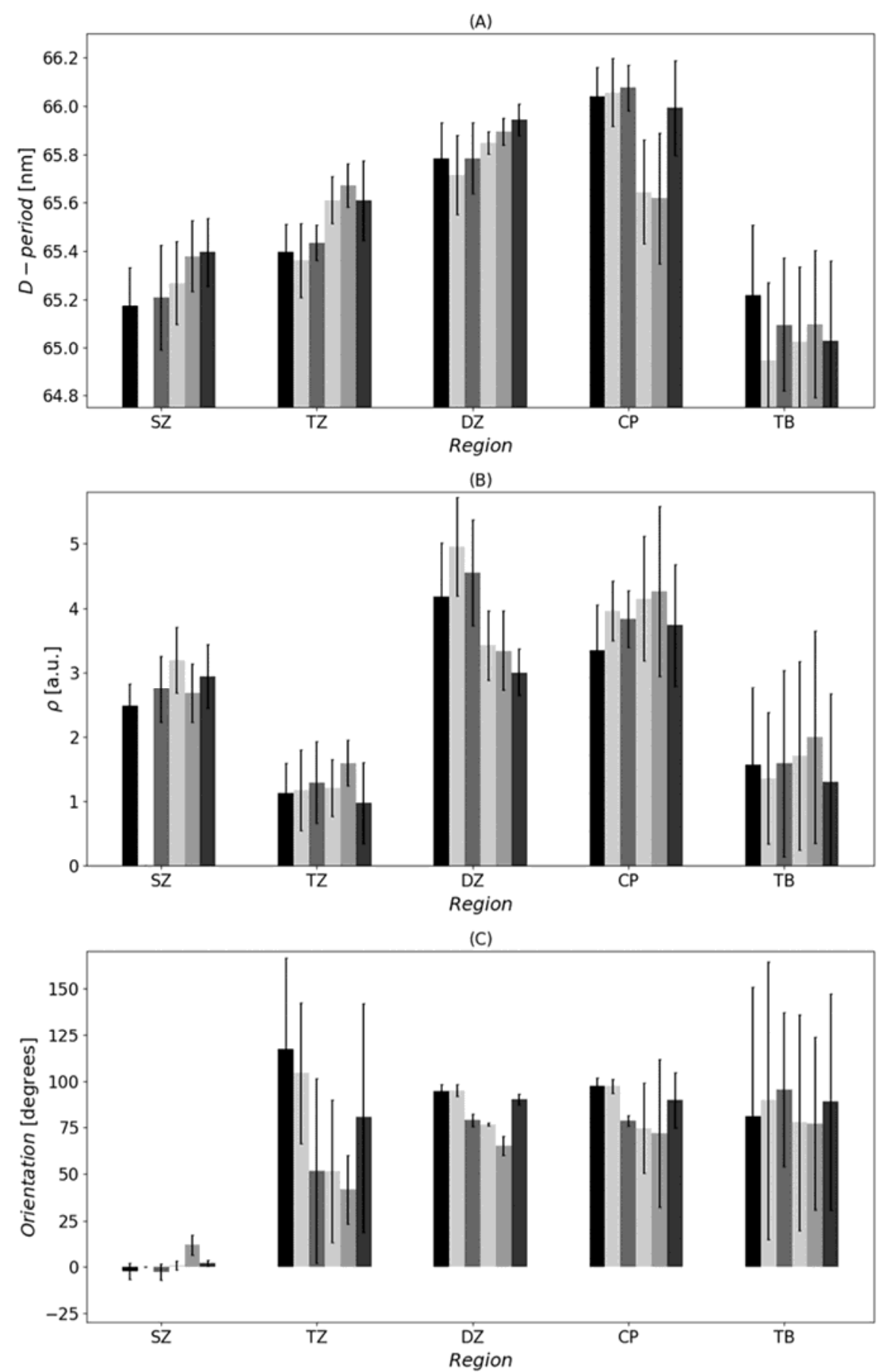

Figure 6

Figure 6: Bar plots for the regular scan $(n=6)$, with error bars representing the standard deviation. (A) D-period, (B) $\rho$ and (C) Fibrillar orientation across the BCU. 

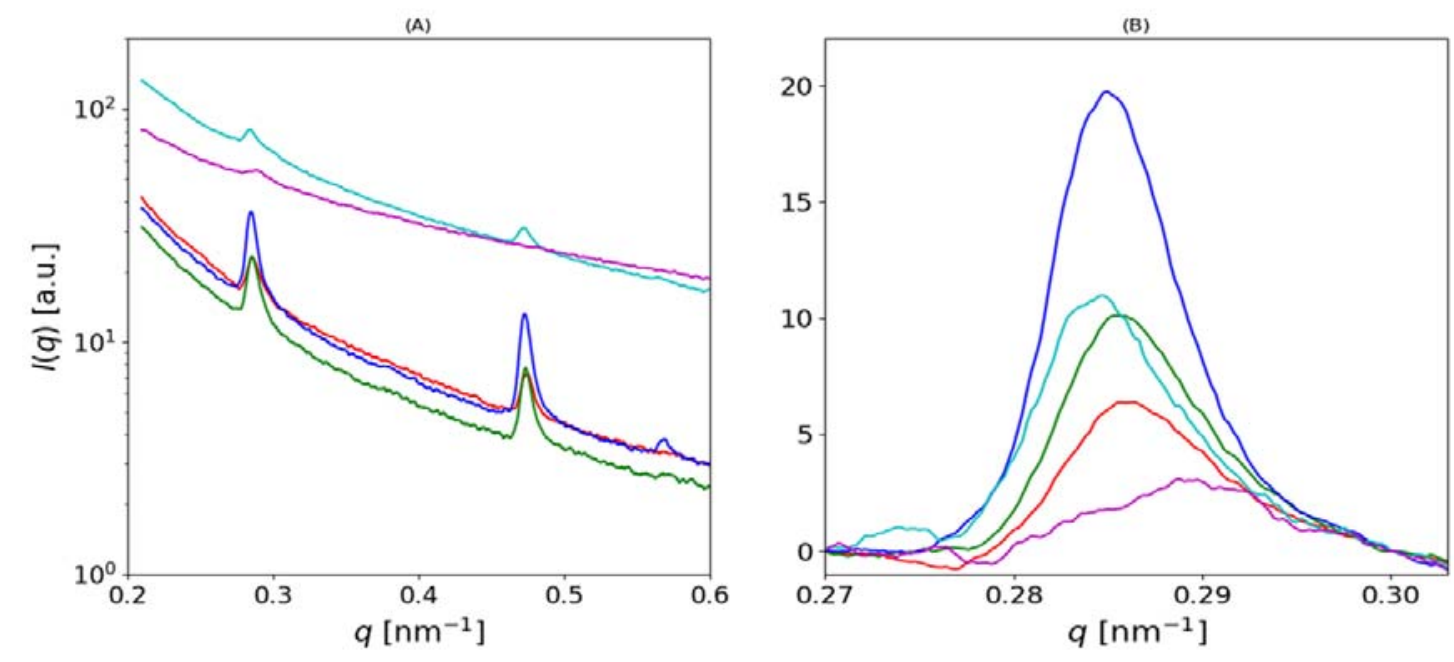

Figure 7

Figure 7: (A) I(q) plot, showing $3^{\text {rd }}$ order and $5^{\text {th }}$ order peaks due to collagen D-period, after azimuthally integration around the diffraction pattern for the q-radial range $0.2-0.6 \mathrm{~nm}^{-1}$. (Red: SZ, Green: TZ, Blue: DZ, Cyan: CP and Magenta: TB). (B) The $3^{\text {rd }}$ order collagen peaks across the BCU after the diffuse SAXS background subtraction. 

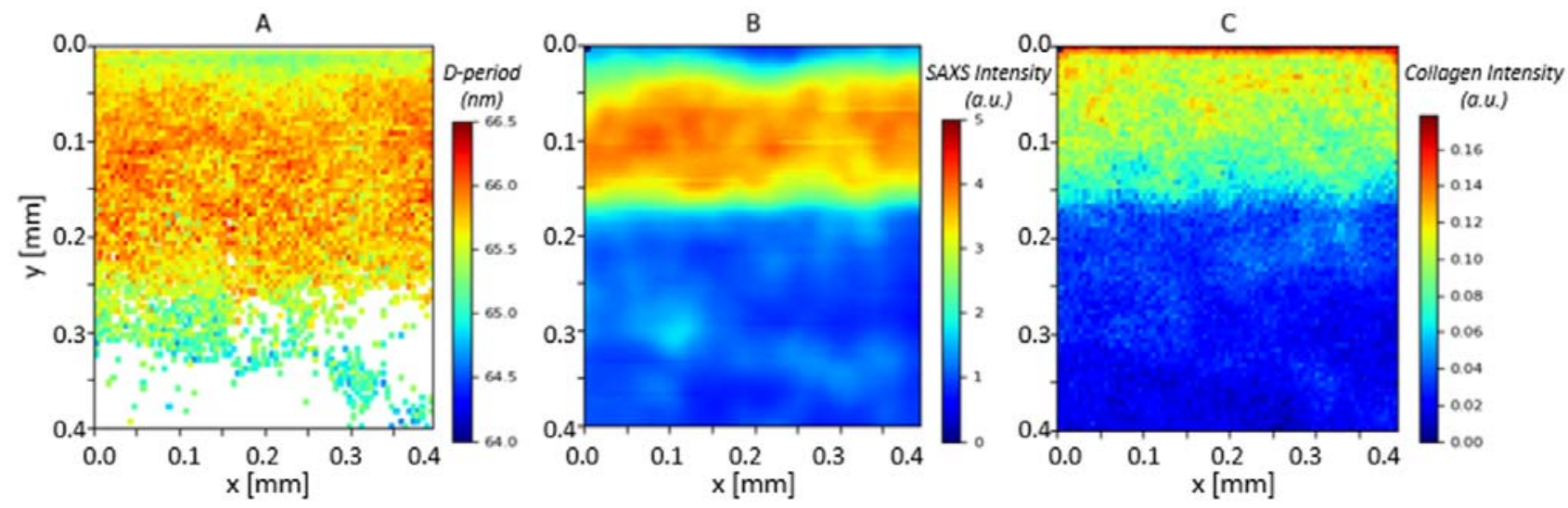

Figure 8

Figure 8: Colour maps for high resolution BCI scans. (A) D-period, (B) SAXS intensity and (C) Collagen intensity, where regions of high intensity correspond with mineral dense region i.e. $\mathrm{CP}$. 

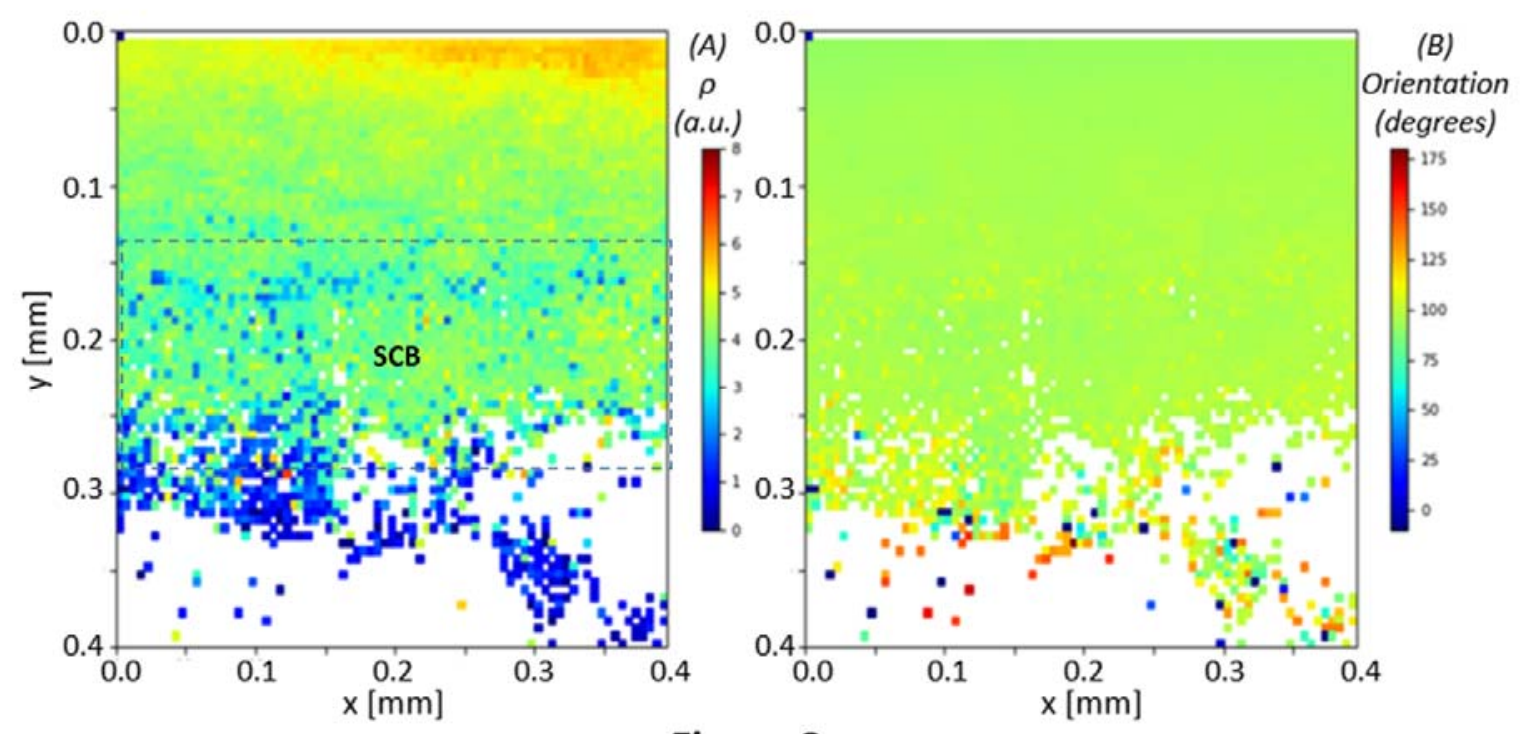

Figure 9

Figure 9: Colour map for the high-resolution scan of BCI for (A) $\rho$ and (B) Collagen fibril orientation. The BCI shows a high degree of fibrillar alignment when transitioning from the $\mathrm{DZ}$ of $\mathrm{AC}$ to the $\mathrm{CP}$, as well as fibres predominantly orientated in the angle of $90^{\circ}$, perpendicular to the articulating surface. This gradient dissipates to lesser degree of fibrillar alignment and more randomly orientated fibre directions in TB. 


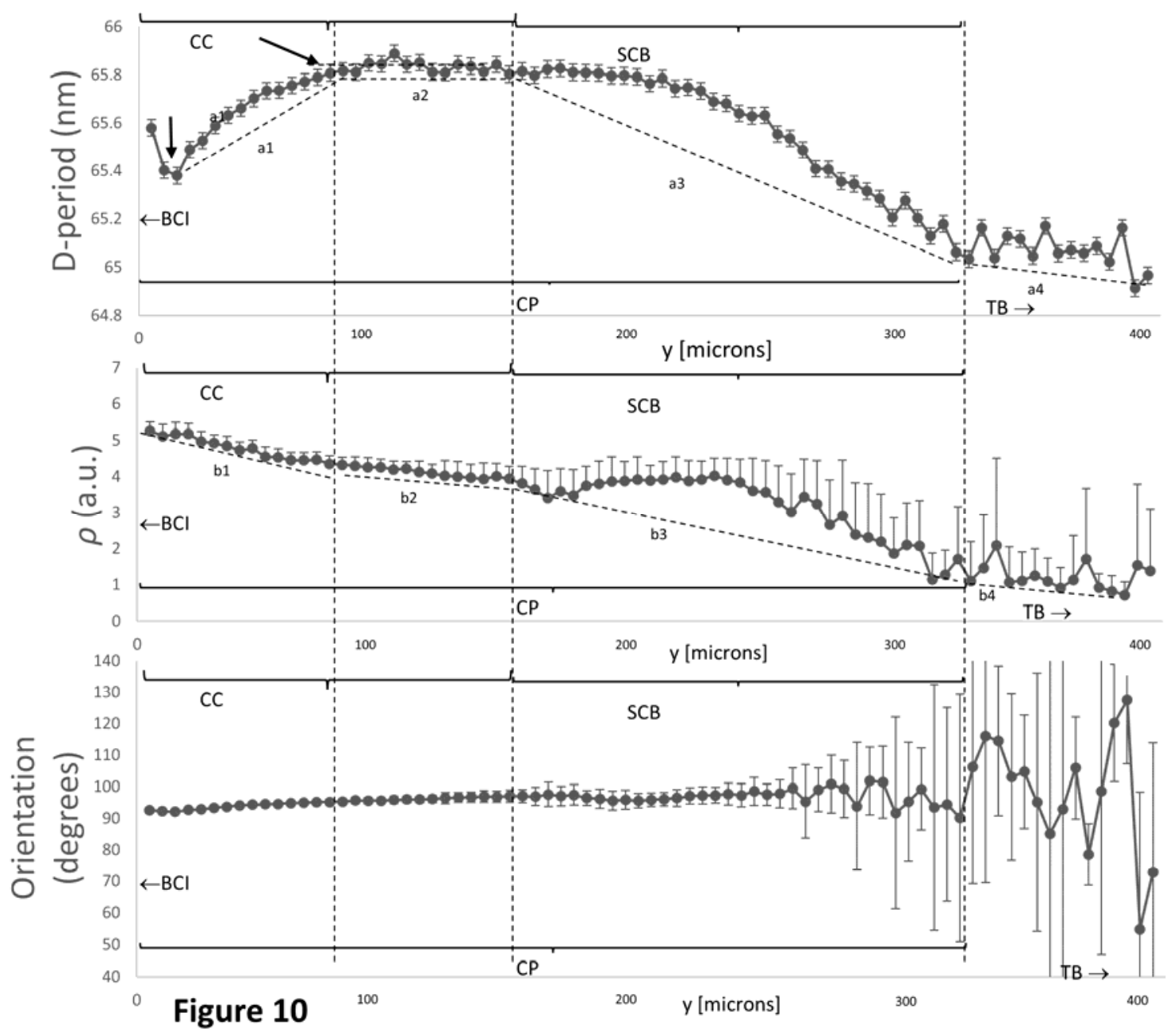

Figure 10: Line graphs for the high resolution BCI scan (A) The mean D-period (B) mean $\rho$ and (C) mean orientation, averaged for each y-coordinate displayed with error bars representing standard error of the mean. Higher D-period and $\rho$ values are found in the CP, with fibrillar orientation around $90^{\circ}$ and more random orientation in $\mathrm{TB}$, which is consistent with findings from the $\mathrm{BCU}$ full length scan. 


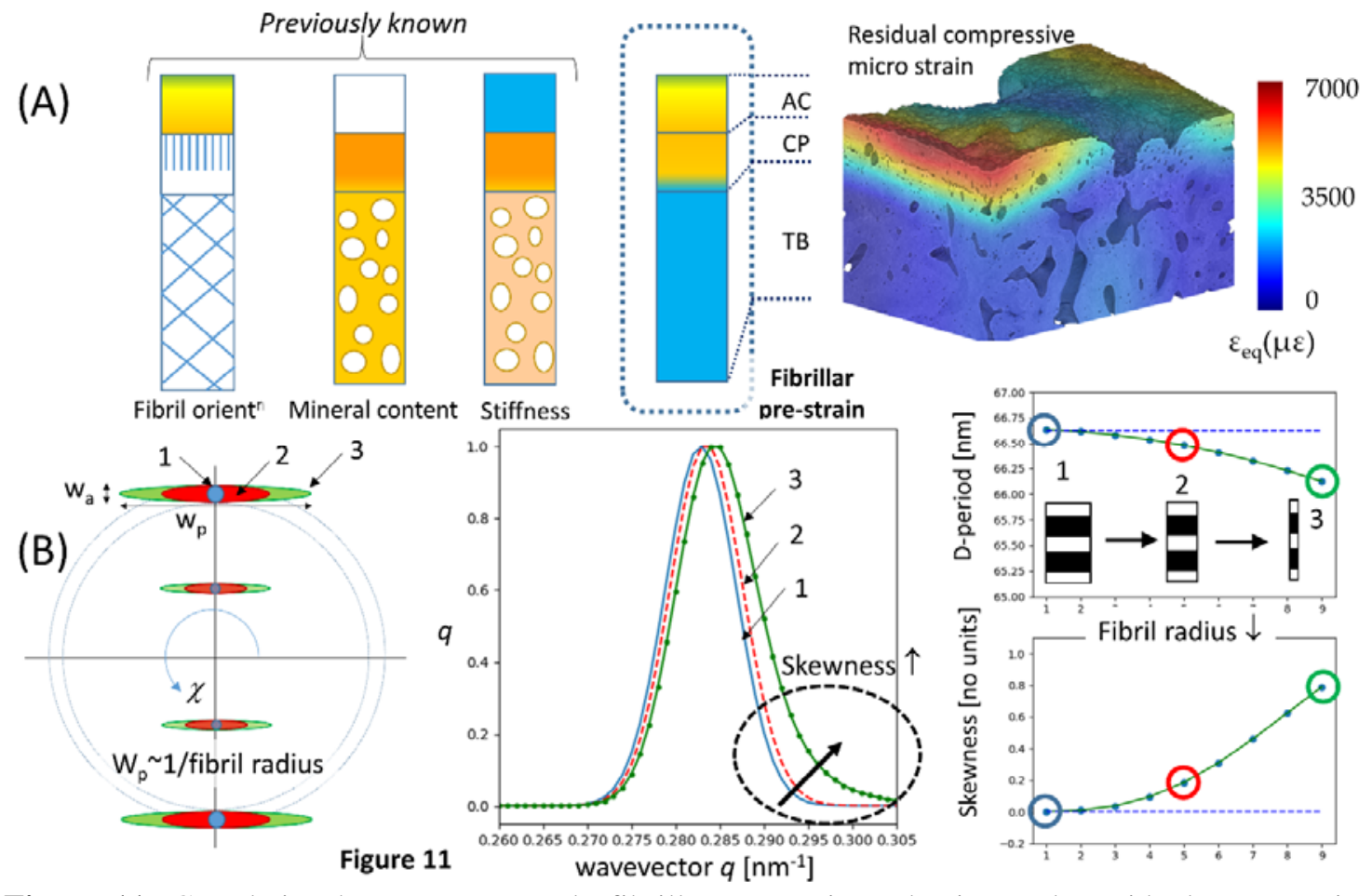

Figure 11: Correlation between nanoscale fibrillar pre-strain and microscale residual compressive strain. Left: schematic of fibrillar pre-strain gradients (this work; Figs 2, 4 and 8) across the AC, CP, and TB in the bovine metacarpophalangeal joint. Right: From independent work on the bovine patellar joint (52), residual compressive strain (after loading) visualised using microcomputed tomography (CT) digital volume correlation (DVC). The calcified plate (both CC and SCB) are seen as regions with both high fibrillar tensile pre-strain (in unloaded state) and high compressive strain (energy storage) after loading. 


\section{Supplementary Materials}

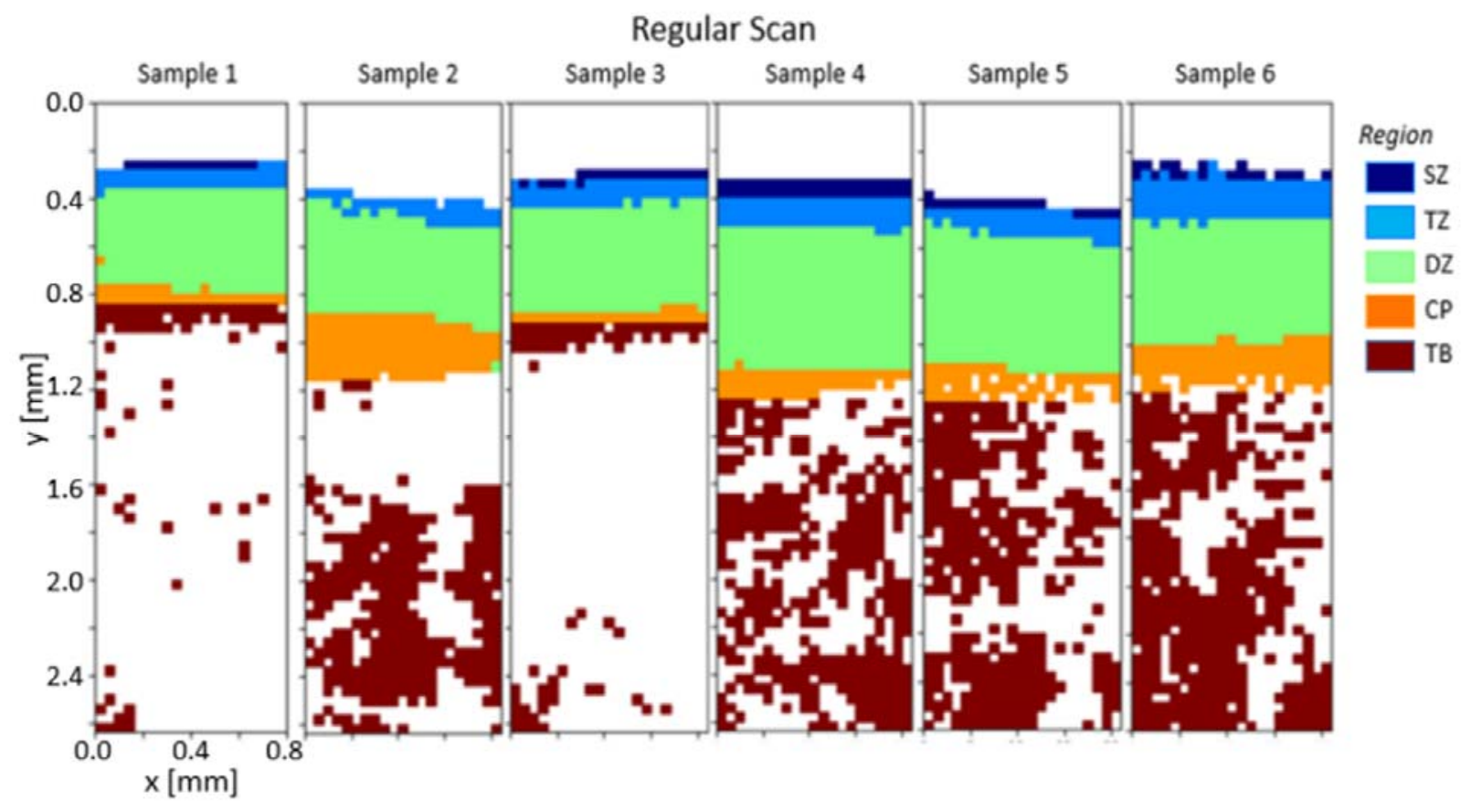

Figure S1

Supplementary Figure 1: Colour plots for the regions across BCU for 6 regular scans.

\begin{tabular}{|c|c|c|c|c|c|c|c|}
\hline \multicolumn{8}{|c|}{ Full-length scan } \\
\hline \multirow{2}{*}{ Parameter } & \multirow{2}{*}{$\begin{array}{l}\text { Anova } \\
\text { P value }\end{array}$} & \multicolumn{6}{|c|}{$\begin{array}{ll}\text { Tukey HSD } \\
\end{array}$} \\
\hline & & Region & Diff & Lwr & Upr & $P$ adj & Signif \\
\hline \multirow{6}{*}{ D-period } & \multirow{6}{*}{$<2 \mathrm{e}-16$} & DZ-CP & -0.1088 & -0.1743 & -0.0434 & 0.0001 & $* * *$ \\
\hline & & TB-CP & -0.9740 & -1.0306 & -0.9174 & 0.0000 & $* * *$ \\
\hline & & TZ-CP & -0.3628 & -0.4471 & -0.2786 & 0.0000 & $* * *$ \\
\hline & & TB-DZ & -0.8651 & -0.9034 & -0.8268 & 0.0000 & $* * *$ \\
\hline & & TZ-DZ & -0.2539 & -0.3272 & -0.1807 & 0.0000 & $* * *$ \\
\hline & & TZ-TB & -0.6111 & 0.5457 & 0.6766 & 0.0000 & $* * *$ \\
\hline \multirow{6}{*}{$\rho$} & \multirow{6}{*}{$<2 \mathrm{e}-16$} & DZ-CP & 0.74466 & -0.8142 & 2.3074 & 0.6080 & $\mathrm{~ns}$ \\
\hline & & TB-CP & -1.9789 & -3.3292 & -0.6284 & 0.0009 & $* * *$ \\
\hline & & TZ-CP & -3.3334 & -5.3429 & -1.3240 & 0.0001 & $* * *$ \\
\hline & & TB-DZ & -2.7255 & -3.6387 & -1.8122 & 0.0000 & $* * *$ \\
\hline & & TZ-DZ & -4.0800 & -5.8259 & -2.3341 & 0.0000 & $* * *$ \\
\hline & & TZ-TB & -1.3545 & -2.9151 & 0.2060 & 0.1151 & $\mathrm{~ns}$ \\
\hline \multirow{6}{*}{ Orientation } & \multirow{6}{*}{0.127} & DZ-CP & -3.1012 & -14.7246 & 8.5220 & 0.9025 & ns \\
\hline & & TB-CP & -5.4598 & -15.5161 & 4.5965 & 0.5021 & ns \\
\hline & & TZ-CP & 3.1326 & -11.8311 & 18.0964 & 0.9497 & ns \\
\hline & & TB-DZ & -2.3585 & -9.1589 & 4.4418 & 0.8092 & ns \\
\hline & & TZ-DZ & 6.2339 & -6.7672 & 19.2350 & 0.6061 & ns \\
\hline & & TZ-TB & 8.5924 & -3.0289 & 20.2138 & 0.2279 & ns \\
\hline
\end{tabular}

Supplementary Table 1: Results from statistical testing of each nanoscale parameter for differences in regions across BCU for full-length scan. ANOVA tests followed by TUKEY HSD tests were carried out on the data. $\mathrm{P}$ values indicate statistical significance, where $* * *$ is $(\mathrm{p}<0.001), * *$ is $(\mathrm{p}<0.01), *$ is $(\mathrm{p}<0.05)$ and $\mathrm{ns}$ is non-significant difference between regions. 


\begin{tabular}{|c|c|c|c|}
\hline \multicolumn{4}{|c|}{ Regular scan } \\
\hline \multirow{2}{*}{ Parameter } & \multirow{2}{*}{ Sample } & Anova & \multirow{2}{*}{ Signif } \\
\hline & & P value & \\
\hline \multirow{6}{*}{ D-period } & 1 & $<2 \mathrm{e}-16$ & $* * *$ \\
\hline & 2 & $<2 \mathrm{e}-16$ & $* * *$ \\
\hline & 3 & $<2 \mathrm{e}-16$ & $* * *$ \\
\hline & 4 & $<2 \mathrm{e}-16$ & $* * *$ \\
\hline & 5 & $<2 \mathrm{e}-16$ & $* * *$ \\
\hline & 6 & $<2 \mathrm{e}-16$ & $* * *$ \\
\hline \multirow{6}{*}{$\rho$} & 1 & $4.88 \mathrm{E}-13$ & $* * *$ \\
\hline & 2 & 4.99E-07 & $* * *$ \\
\hline & 3 & $5.05 \mathrm{E}-16$ & $* * *$ \\
\hline & 4 & 0.167 & ns \\
\hline & 5 & 0.00306 & $* *$ \\
\hline & 6 & 0.000191 & $* * *$ \\
\hline \multirow{6}{*}{ Orientation } & 1 & $<2 \mathrm{e}-16$ & $* * *$ \\
\hline & 2 & 0.121 & $\mathrm{~ns}$ \\
\hline & 3 & $<2 \mathrm{e}-16$ & $* * *$ \\
\hline & 4 & $<2 \mathrm{e}-16$ & $* * *$ \\
\hline & 5 & $<2 \mathrm{e}-16$ & $* * *$ \\
\hline & 6 & $1.45 \mathrm{E}-15$ & $* * *$ \\
\hline
\end{tabular}

Supplementary Table 2: Results from statistical testing of each nanoscale parameter for differences in regions across $6 \mathrm{BCU}$ for regular scan. ANOVA test was carried out on the data. $\mathrm{P}$ values indicate statistical significance, where $* * *$ is $(\mathrm{p}<0.001), * *$ is $(\mathrm{p}<0.01), *$ is $(\mathrm{p}<0.05)$ and $\mathrm{ns}$ is non-significant difference between regions. 


\section{References}

1. Goldring SR, Goldring MB. Changes in the osteochondral unit during osteoarth ritis: Structure, function and cartilage bone crosstalk. Nature Reviews Rheumatology. 2016.

2. Yuan XL, Meng HY, Wang YC, Peng J, Guo QY, Wang AY, et al. Bone-cartilage interface crosstalk in osteoarthritis: Potential pathways and future therapeutic strategies. Vol. 22, Osteoarthritis and Cartilage. 2014. p. 1077-89.

3. James SL, Abate D, Abate KH, Abay SM, Abbafati C, Abbasi N, et al. Global, regional, and national incidence, prevalence, and years lived with disability for 354 Diseases and Injuries for 195 countries and territories, 1990-2017: A systematic analysis for the Global Burden of Disease Study 2017. Lancet. 2018;392(10159).

4. Cui A, Li H, Wang D, Zhong J, Chen Y, Lu H. Global, regional prevalence, incidence and risk factors of knee osteoarthritis in population-based studies. EClinicalMedicine. 2020;29-30.

5. Mobasheri A, Henrotin Y. Biomarkers of (osteo)arthritis. Vol. 20, Biomarkers. 2015. p. 513-8.

6. Liu Z, Meyers MA, Zhang Z, Ritchie RO. Functional gradients and heterogeneities in biological materials: Design principles, functions, and bioinspired applications. Prog Mater Sci. 2017;88:467-98.

7. Benninghoff A. Form und Bau der Gelenkknorpel in ihren Beziehungen zur Funktion. Zeitschrift für Zellforsch und Mikroskopische Anat. 1925;2(5):783-862.

8. Lu XL, Mow VC. Biomechanics of articular cartilage and determination of material properties. Medicine and Science in Sports and Exercise. 2008.

9. Inamdar SR, Barbieri E, Terrill NJ, Knight MM, Gupta HS. Proteoglycan degradation mimics static compression by altering the natural gradients in fibrillar organisation in cartilage. Acta Biomater. 2019;97:437-50.

10. Zizak I, Roschger P, Paris O, Misof BM, Berzlanovich A, Bernstorff S, et al. Characteristics of mineral particles in the human bone/cartilage interface. J Struct Biol. 2003;141(3):208-17.

11. Finnilä MAJ, Gupta S Das, Turunen MJ, Kestilä I, Turkiewicz A, Lutz-Bueno V, et al. Mineral crystal thickness in calcified cartilage and subchondral bone in healthy and osteoarthritic knees. bioRxiv [Internet]. 2021 Jun;2021.06.15.448181. Available from:

http://biorxiv.org/cgi/content/short/2021.06.15.448181v1?rss=1\&utm_source=researcher_a pp\&utm_medium=referral\&utm_campaign=RESR_MRKT_Researcher_inbound

12. Gupta HSS, Schratter S, Tesch W, Roschger P, Berzlanovich A, Schoeberl T, et al. Two different correlations between nanoindentation modulus and mineral content in the bone-cartilage interface. J Struct Biol. 2005;149(2). 
13. Weiner S, Traub W. Bone structure: from ångstroms to microns. FASEB J. 1992;

14. Madi K, Staines KA, Bay BK, Javaheri B, Geng H, Bodey AJ, et al. In situ characterization of nanoscale strains in loaded whole joints via synchrotron X-ray tomography. Nat Biomed Eng. 2020;4:343-354.

15. Burr DB, Radin EL. Microfractures and microcracks in subchondral bone: Are they relevant to osteoarthrosis? Rheumatic Disease Clinics of North America. 2003.

16. Fratzl P, Weinkamer R. Nature's hierarchical materials. Progress in Materials Science. 2007.

17. Sophia Fox AJ, Bedi A, Rodeo SA. The basic science of articular cartilage: structure, composition, and function. Sports Health. 2009;1(6):461-8.

18. Inamdar SR, Knight DP, Terrill NJ, Karunaratne A, Cacho-Nerin F, Knight MM, et al. The Secret Life of Collagen: Temporal Changes in Nanoscale Fibrillar Pre-Strain and Molecular Organization during Physiological Loading of Cartilage. ACS Nano. 2017;11(10):9728-37.

19. Tadimalla S, Tourell MC, Knott KI, Momot KI, Knott R, Momot KI. Quantifying collagen fibre architecture in articular cartilage using small-angle X-ray scattering. Biomed Spectrosc Imaging. 2017;6(May):37-57.

20. Fang M, Goldstein EL, Turner AS, Les CM, Orr BG, Fisher GJ, et al. Type i collagen D-spacing in fibril bundles of dermis, tendon, and bone: Bridging between nano- and micro-level tissue hierarchy. ACS Nano. 2012;

21. Zimmermann EA, Schaible E, Bale H, Barth HD, Tang SYY, Reichert P, et al. Age-related changes in the plasticity and toughness of human cortical bone at multiple length scales. Proc Natl Acad Sci U S A. 2011;108(35):14416-21.

22. Bell JS, Hayes S, Whitford C, Sanchez-Weatherby J, Shebanova O, Vergari C, et al. The hierarchical response of human corneal collagen to load. Acta Biomater. 2018;65:216-25.

23. Gautieri A, Passini FS, Silvan U, Guizar-Sicairos M, Carimati G, Volpi P, et al. Advanced glycation end-products: Mechanics of aged collagen from molecule to tissue. Matrix Biol. 2016;

24. Leighton MP, Rutenberg AD, Kreplak L. D-band strain underestimates fibril strain for twisted collagen fibrils at low strains. J Mech Behav Biomed Mater. 2021;

25. Bhosale AM, Richardson JB. Articular cartilage: structure, injuries and review of management. Br Med Bull. 2008 Jan;87:77-95.

26. Masic A, Bertinetti L, Schuetz R, Chang S-W, Metzger TH, Buehler MJ, et al. Osmotic Pressure Induced Tensile Forces in Tendon Collagen. Nat Commun. 2015;6:5942.

27. Fang W, Ping H, Wagermaier W, Jin S, Amini S, Fratzl P, et al. Rapid collagen-directed mineralization of calcium fluoride nanocrystals with periodically patterned nanostructures. 
Nanoscale. 2021;

28. Lees S. Mineralization of type I collagen. Biophys J. 2003;

29. Temple DK, Cederlund AA, Lawless BM, Aspden RM, Espino DM. Viscoelastic properties of human and bovine articular cartilage: a comparison of frequency-dependent trends. BMC Musculoskelet Disord. 2016;

30. Burgin LV., Aspden RM. Impact testing to determine the mechanical properties of articular cartilage in isolation and on bone. J Mater Sci Mater Med. 2008;

31. Smith AJ, Alcock SG, Davidson LS, Emmins JH, Hiller Bardsley JC, Holloway P, et al. 122: SAXS/WAXS beamline at Diamond Light Source - an overview of 10 years operation. J Synchrotron Radiat. 2021;28:939-47.

32. Henrich B, Bergamaschi A, Broennimann C, Dinapoli R, Eikenberry EF, Johnson I, et al. PILATUS: A single photon counting pixel detector for X-ray applications. Nucl Instruments Methods Phys Res Sect A Accel Spectrometers, Detect Assoc Equip. 2009;607(1).

33. Filik J, Ashton AW, Chang PCY, Chater PA, Day SJ, Drakopoulos M, et al. Processing twodimensional X-ray diffraction and small-angle scattering data in DAWN 2. J Appl Crystallogr. 2017 Jun 1;50(3):959-66.

34. Gourrier A, Wagermaier W, Burghammer M, Lammie D, Gupta HS, Fratzl P, et al. Scanning Xray imaging with small-angle scattering contrast. J Appl Crystallogr. 2007;40(SUPPL. 1).

35. Blowes LM, Egertová M, Liu Y, Davis GR, Terrill NJ, Gupta HS, et al. Body wall structure in the starfish Asterias rubens. J Anat. 2017;231(3).

36. Liebi M, Georgiadis M, Menzel A, Schneider P, Kohlbrecher J, Bunk O, et al. Nanostructure surveys of macroscopic specimens by small-angle scattering tensor tomography. Nature. 2015;527:349-352.

37. Fratzl P. Collagen: Structure and Mechanics. Fratzl P, editor. Collagen: Structure and Mechanics. Springer US; 2008. 1-506 p.

38. Xi L, De Falco P, Barbieri E, Karunaratne A, Bentley L, Esapa CT, et al. Bone matrix development in steroid-induced osteoporosis is associated with a consistently reduced fibrillar stiffness linked to altered bone mineral quality. Acta Biomater. 2018;76.

39. Yang W, Sherman VR, Gludovatz B, Schaible E, Stewart P, Ritchie RO, et al. On the tear resistance of skin. Nat Commun. 2015;6:6649.

40. Almer JD, Stock SR. Internal strains and stresses measured in cortical bone via high-energy Xray diffraction. J Struct Biol. 2005;152(1):14-27.

41. Xi L, Zhang Y, Gupta H, Terrill N, Wang P, Zhao T, et al. A multiscale study of structural and compositional changes in a natural nanocomposite: Osteoporotic bone with chronic 
endogenous steroid excess. Bone. 2020;

42. Newville M, Otten R, Nelson A, Ingargiola A, Stensitzki T, Allan D, et al. Imfit/Imfit-py 1.0.2 [Internet]. Zenodo; 2021. Available from: https://doi.org/10.5281/zenodo.4516651

43. Karunaratne A, Xi L, Bentley L, Sykes D, Boyde A, Esapa CT, et al. Multiscale alterations in bone matrix quality increased fragility in steroid induced osteoporosis. Bone. 2016;84:15-24.

44. Xi L, De Falco P, Barbieri E, Karunaratne A, Bentley L, Esapa CT, et al. Reduction of fibrillar strain-rate sensitivity in steroid-induced osteoporosis linked to changes in mineralized fibrillar nanostructure. Bone. 2020;131.

45. Hunter JD. Matplotlib: A 2D graphics environment. Comput Sci Eng. 2007;9(3):90-5.

46. Pabisch S, Wagermaier W, Zander T, Li C, Fratzl P. Chapter Eighteen - Imaging the Nanostructure of Bone and Dentin Through Small- and Wide-Angle X-Ray Scattering. In: De Yoreo JJBT-M in E, editor. Research Methods in Biomineralization Science. Academic Press; 2013. p. 391-413.

47. FratzI P, Misof K, Zizak I, Rapp G, Bernstorff S, Amenitsch H. Fibrillar Structure and Mechanical Properties of Collagen. J Struct Biol. 1997 Jan;122(1-2):119-22.

48. Fratzl P, Fratzl-Zelman N, Klaushofer K. Collagen packing and mineralization. An x-ray scattering investigation of turkey leg tendon. Biophys J. 1993;

49. Guinier A, Fournet G. Small-Angle Scattering of X-rays. John Wiley \& Sons Ltd.; 1955.

50. Khanarian NT, Boushell MK, Spalazzi JP, Pleshko N, Boskey AL, Lu HH. FTIR-I compositional mapping of the cartilage-to-bone interface as a function of tissue region and age. J Bone Miner Res. 2014;

51. Moger CJ, Barrett R, Bleuet P, Bradley DA, Ellis RE, Green EM, et al. Regional variations of collagen orientation in normal and diseased articular cartilage and subchondral bone determined using small angle X-ray scattering (SAXS). Osteoarthr Cartil. 2007 Jun;15(6):6827.

52. Tozzi G, Fernández MP, Davis S, Karali A, Kao AP, Blunn G. Full-field strain uncertainties and residuals at the cartilage-bone interface in unstained tissues using propagation-based phasecontrast XCT and digital volume correlation. Materials (Basel). 2020;

53. Liebi M, Georgiadis M, Kohlbrecher J, Holler M, Raabe J, Usov I, et al. Small-angle X-ray scattering tensor tomography: Model of the three-dimensional reciprocal-space map, reconstruction algorithm and angular sampling requirements. Acta Crystallogr Sect A Found Adv. 2018;A74:12-24.

54. Giannini C, De Caro L, Terzi A, Fusaro L, Altamura D, Diaz A, et al. Decellularized pericardium tissues at increasing glucose, galactose and ribose concentrations and at different time 

available under aCC-BY 4.0 International license.

points studied using scanning X-ray microscopy. IUCrJ. 2021 Jul;8(Pt 4):621-32.

55. Qin HJ, Xu T, Wu HT, Yao ZL, Hou YL, Xie YH, et al. SDF-1/CXCR4 axis coordinates crosstalk between subchondral bone and articular cartilage in osteoarthritis pathogenesis. Bone. 2019;125.

56. Findlay DM, Kuliwaba JS. Bone-cartilage crosstalk: A conversation for understanding osteoarthritis. Bone Research. 2016. 